Pacific

Journal of

Mathematics

\title{
DRINFELD ORBIFOLD ALGEBRAS
}

ANNE V. SHEPLER AND SARAH WitherSPOON

Volume 259 No. 1

September 2012 


\title{
DRINFELD ORBIFOLD ALGEBRAS
}

\author{
ANNE V. SHEPLER AND SARAH WITHERSPOON
}

\begin{abstract}
We define Drinfeld orbifold algebras as filtered algebras deforming the skew group algebra (semidirect product) arising from the action of a finite group on a polynomial ring. They simultaneously generalize Weyl algebras, graded (or Drinfeld) Hecke algebras, rational Cherednik algebras, symplectic reflection algebras, and universal enveloping algebras of Lie algebras with group actions. We give necessary and sufficient conditions on defining parameters to obtain Drinfeld orbifold algebras in two general formats, both algebraic and homological. Our algebraic conditions hold over any field of characteristic other than two, including fields whose characteristic divides the order of the acting group. We explain the connection between Hochschild cohomology and a Poincaré-Birkhoff-Witt property explicitly (using Gerstenhaber brackets). We also classify those deformations of skew group algebras which arise as Drinfeld orbifold algebras and give applications for abelian groups.
\end{abstract}

\section{Introduction}

Results in commutative algebra are often obtained by an excursion through a larger, noncommutative universe. Indeed, interesting noncommutative algebras often arise from deforming the relations of a classical commutative algebra. Noncommutative algebras modeled on groups acting on commutative polynomial rings serve as useful tools in representation theory and combinatorics, for example, and include symplectic reflection algebras, rational Cherednik algebras, and Lusztig's graded affine Hecke algebras. These algebras are deformations of the skew group algebra generated by a finite group and a polynomial ring (upon which the group acts). They also provide an algebraic framework for understanding geometric deformations of orbifolds.

The first author was partially supported by NSF grants DMS-0800951 and DMS-1101177 and a research fellowship from the Alexander von Humboldt Foundation. The second author was partially supported by NSF grants DMS-0800832 and DMS-1101399.

MSC2010: 16E40, 16S35, 16S80, 16W70, $20 \mathrm{C} 08$.

Keywords: Hochschild cohomology, deformations, skew group algebra, symplectic reflection algebra, graded Hecke algebra. 
Let $G$ be a finite group acting by linear transformations on a finite dimensional vector space $V$ over a field $k$. Let $S:=S(V)$ be the symmetric algebra with the induced action of $G$ by automorphisms, and let $S \# G$ be the corresponding skew group algebra. A graded Hecke algebra (often called a Drinfeld Hecke algebra) emerges after deforming the relations of the symmetric algebra $S$ inside $S \# G$ : We set each expression $x y-y x$ (for $x, y$ in $V$ ) in the tensor algebra $T(V)$ equal to an element of the group ring $k G$ and consider the quotient of $T(V) \# G$ by such relations. The quotient is a deformation of $S \# G$ when the relations satisfy certain conditions, and these conditions are explored in many papers (see, e.g., [Drinfeld 1986; Etingof and Ginzburg 2002; Griffeth 2010; Lusztig 1989; Ram and Shepler 2003]).

Symplectic reflection algebras are special cases of graded Hecke algebras which generalize Weyl algebras in the context of group actions on symplectic spaces. In this paper, we replace Weyl algebras with universal enveloping algebras of Lie algebras and complete the analogy: Weyl algebras are to symplectic reflection algebras as universal enveloping algebras are to what? Our answer is the class of Lie orbifold algebras, which together with graded Hecke algebras belong to a larger class of Drinfeld orbifold algebras as we define and explore in this article.

In [Shepler and Witherspoon 2008], we explained that graded Hecke algebras are precisely those deformations of $S \# G$ which arise from Hochschild 2-cocycles of degree zero with respect to a natural grading on cohomology. In fact, we showed that every such cocycle defines a graded Hecke algebra and thus lifts to a deformation of $S \# G$. The present investigation is partly motivated by a desire to understand deformations of $S \# G$ arising from Hochschild 2-cocycles of degree 1.

Specifically, we assign degree 1 to each $v$ in $V$ and degree 0 to each $g$ in $G$ and consider the corresponding grading on $T(V) \# G$. We set each expression $x \otimes y-y \otimes x$ in the tensor algebra $T(V)$ equal to an element of degree at most 1 (i.e., nonhomogeneous of filtered degree 1) and consider the quotient of $T(V) \# G$ by these relations as a filtered algebra. We call the resulting algebra a Drinfeld orbifold algebra if it satisfies the Poincaré-Birkhoff-Witt property, i.e., if its associated graded algebra is isomorphic to $S \# G$. Such algebras were studied by Halbout, Oudom, and Tang [Halbout et al. 2011] over the real numbers in the special case that $G$ acts faithfully. We give a direct algebraic approach for arbitrary group actions and fields here (which includes the case when the characteristic of the field divides the order of $G$ ).

In this article, we explain in detail the connections between the Poincaré-BirkhoffWitt property, deformation theory, and Hochschild cohomology. We first classify those deformations of $S \# G$ which arise as Drinfeld orbifold algebras. We then derive necessary and sufficient conditions on algebra parameters that should facilitate efforts to study and classify these algebras. In particular, we express the PBW 
property as a set of conditions using the diamond lemma. (Although our conditions hold over arbitrary characteristic, we include a comparison with the theory of Koszul rings over $k G$ used in [Etingof and Ginzburg 2002; Halbout et al. 2011], which requires $k G$ to be semisimple.) We give an explicit road map from cohomology, expressed in terms of Koszul resolutions, to the defining relations for Drinfeld orbifold algebras. In particular, we explain how PBW conditions enjoy an elegant description in terms of Gerstenhaber brackets.

Note that one can not automatically deduce results for Drinfeld orbifold algebras defined over $\mathbb{C}$ from the results in [Halbout et al. 2011] for similar algebras defined over $\mathbb{R}$. (For example, the infinitesimal of a nontrivial deformation over $\mathbb{C}$ associated to a group $G$ acting on a complex vector space is always supported off the set $\mathscr{R}$ of complex reflections in $G$, yet the infinitesimal of a nontrivial deformation over $\mathbb{R}$ associated to that same group (acting on a real vector space of twice the dimension) may have support including $\mathscr{R}$.)

More precisely, let us consider a linear "parameter" function mapping the exterior product $V \wedge V$ to that part of $T(V) \# G$ having degree at most 1:

$$
\kappa: V \wedge V \rightarrow(k \oplus V) \otimes k G .
$$

We drop the tensor sign when expressing elements of $T(V)$ and $T(V) \# G$ (as is customary when working with noncommutative, associative algebras), writing $v w$ in place of $v \otimes w$, for example. We also usually write $\kappa(v, w)$ for $\kappa(v \wedge w)$, to make some complicated expressions clearer. Define an algebra $\mathscr{H}:=\mathscr{H}_{\kappa}$ as the quotient

$$
\mathscr{H}_{\kappa}:=T(V) \# G /(v w-w v-\kappa(v, w) \mid v, w \in V) .
$$

We say that $\mathscr{H}_{\kappa}$ satisfies the $P B W$ condition when its associated graded algebra gr $\mathscr{H}_{\kappa}$ is isomorphic to $S \# G$ (in analogy with the Poincaré-Birkhoff-Witt Theorem for universal enveloping algebras). In this case, we call $\mathscr{H}_{\kappa}$ a Drinfeld orbifold algebra. One may check that the PBW condition is equivalent to the existence of a basis

$$
\left\{v_{1}^{m_{1}} \cdots v_{n}^{m_{n}} g: m_{i} \in \mathbb{Z}_{\geq 0}, g \in G\right\}
$$

for $\mathscr{H}_{\kappa}$ as a $k$-vector space, where $v_{1}, \ldots, v_{n}$ is a $k$-basis of $V$.

The terminology arises because Drinfeld [1986] first considered deforming the algebra of coordinate functions $S^{G}$ of the orbifold $V^{*} / G$ (over $\mathbb{C}$ ) in this way, although his original construction required the image of $\kappa$ to lie in the group algebra $\mathbb{C} G$. Indeed, when $\kappa$ has image in $k G$, a Drinfeld orbifold algebra $\mathscr{H}_{\kappa}$ is called a Drinfeld Hecke algebra. These algebras are also called graded Hecke algebras, as the graded affine Hecke algebra defined by Lusztig [1988; 1989] is a special case (arising when $G$ is a Coxeter group; see [Ram and Shepler 2003, Section 3]). Note that symplectic reflection algebras are also examples of these algebras. 
Drinfeld orbifold algebras compose a large class of deformations of the skew group algebra $S \# G$, as explained in this paper. We determine necessary and sufficient conditions on $\kappa$ so that $\mathscr{H}_{\kappa}$ satisfies the PBW condition and interpret these conditions in terms of Hochschild cohomology. To illustrate, we give several small examples in Sections 3 and 4. We show that a special case of this construction is a class of deformations of the skew group algebras $U \# G$, where $U$ is the universal enveloping algebra of a finite dimensional Lie algebra upon which $G$ acts. These deformations are termed Lie orbifold algebras.

For example, consider the Lie algebra $\mathfrak{s l}_{2}$ of $2 \times 2$ matrices over $\mathbb{C}$ having trace 0 with usual basis $e, f, h$. A cyclic group $G$ of order 2 generated by $g$ acts as follows: ${ }^{g} e=f,{ }^{g} f=e,{ }^{g} h=-h$. Let $V$ be the underlying $\mathbb{C}$-vector space of $\mathfrak{s l}_{2}$ and consider the quotient

$$
T(V) \# G /(e h-h e+2 e-g, h f-f h+2 f-g, e f-f e-h) .
$$

We show in Example 4.3 that this quotient is a Lie orbifold algebra. Notice that if we delete the degree 0 term (that is, the group element $g$ ) in each of the first two relations above, we obtain the skew group algebra $U\left(\mathfrak{s l}_{2}\right) \# G$. If we delete the degree 1 terms instead, we obtain a Drinfeld Hecke algebra (i.e., graded Hecke algebra). (This is a general property of Lie orbifold algebras that we make precise in Proposition 4.1.)

We assume throughout that $k$ is a field whose characteristic is not 2. For our homological results in Sections 5 through 8, we require in addition that the order of $G$ is invertible in $k$ and that $k$ contains all eigenvalues of the actions of elements of $G$ on $V$; this assumption is not needed for the first few sections. All tensor products will be over $k$ unless otherwise indicated.

\section{Deformations of skew group algebras}

Before exploring necessary and sufficient conditions for an arbitrary quotient algebra to define a Drinfeld orbifold algebra, we explain the connection between these algebras and deformations of the skew group algebra $S \# G$. Recall that $S \# G$ is the $k$ vector space $S \otimes k G$ with algebraic structure given by $\left(s_{1} \otimes g\right)\left(s_{2} \otimes h\right)=s_{1}{ }^{g}\left(s_{2}\right) \otimes g h$ for all $s_{i}$ in $S$ and $g, h$ in $G$. Here, ${ }^{g} s$ denotes the element resulting from the group action of $g$ on $s$ in $S$. Recall that we drop the tensor symbols and simply write, for example, $s_{1} g s_{2} h=s_{1}{ }^{g} s_{2} g h$. We show in the next theorem how Drinfeld orbifold algebras arise as a special class of deformations of $S \# G$.

First, we recall some standard notation. Let $R$ be any algebra over the field $k$, and let $t$ be an indeterminate. A deformation of $R$ over $k[t]$ is an associative $k[t]$-algebra with underlying vector space $R[t]$ and multiplication determined by

$$
r * s=r s+\mu_{1}(r \otimes s) t+\mu_{2}(r \otimes s) t^{2}+\cdots
$$


for all $r, s \in R$, where $r s$ is the product of $r$ and $s$ in $R$, the $\mu_{i}: R \otimes R \rightarrow R$ are $k$-linear maps that are extended to be $k[t]$-linear, and the above sum is finite for each $r, s$.

We adapt our definition of $\mathscr{H}_{\kappa}$ to that of an algebra over $k[t]$. First, decompose $\kappa$ into its constant and linear parts: Let

$$
\kappa=\kappa^{C}+\kappa^{L}, \quad \text { where } \quad \kappa^{C}: V \wedge V \rightarrow k G, \quad \kappa^{L}: V \wedge V \rightarrow V \otimes k G .
$$

Write

$$
\kappa=\sum_{g \in G} \kappa_{g} g
$$

where each (alternating, bilinear) map $\kappa_{g}: V \times V \rightarrow k \oplus V$ also decomposes into constant and linear parts:

$$
\kappa_{g}=\kappa_{g}^{C}+\kappa_{g}^{L}, \quad \text { where } \quad \kappa_{g}^{C}: V \wedge V \rightarrow k, \quad \kappa_{g}^{L}: V \wedge V \rightarrow V .
$$

Now let

$$
\mathscr{H}_{\kappa, t}:=T(V) \# G[t] /\left(v w-w v-\kappa^{L}(v, w) t-\kappa^{C}(v, w) t^{2} \mid v, w \in V\right) .
$$

We call $\mathscr{H}_{\kappa, t}$ a Drinfeld orbifold algebra over $k[t]$ whenever $\mathscr{H}_{\kappa}$ is a Drinfeld orbifold algebra; in this case, $\mathscr{H}_{\kappa, t}$ is a deformation of $S \# G$ over $k[t]$ and $\mathscr{H}_{\kappa, t} / t \mathscr{H}_{\kappa, t} \cong S \# G$.

The following theorem extends [Witherspoon 2007, Theorem 3.2] (in the case of a trivial twisting cocycle) to our setting. We note that in case $\kappa^{L} \equiv 0$, a change of formal parameter allows us to replace $t^{2}$ by $t$ in the definition of $\mathscr{H}_{\kappa, t}$, thus giving the Drinfeld Hecke algebras (i.e., graded Hecke algebras) over $k[t]$ (defined in [Witherspoon 2007]) as a special case. We use standard notation for graded linear maps: If $W$ and $W^{\prime}$ are graded vector spaces, a linear map $\alpha: W \rightarrow W^{\prime}$ is homogeneous of degree $\operatorname{deg} \alpha$ if $\alpha\left(W_{i}\right) \subseteq W_{i+\operatorname{deg} \alpha}$ for all $i$.

Theorem 2.1. The Drinfeld orbifold algebras $\mathscr{H}_{\kappa, t}$ over $k[t]$ are precisely the deformations of $S \# G$ over $k[t]$ for which $\operatorname{deg} \mu_{i}=-i$ and for which $k G$ is in the kernel of $\mu_{i}$ for all $i \geq 1$.

The hypothesis that $k G$ is in the kernel of all $\mu_{i}$ is a reasonable one when the characteristic of $k$ does not divide the order of $G$ : In this case one may choose to work with maps that are linear over the semisimple ground ring $k G$ as in [Beilinson et al. 1996; Etingof and Ginzburg 2002]. There are however alternative ways to express Drinfeld Hecke algebras for which this hypothesis is not true. See [Ram and Shepler 2003, Theorem 3.5] for a comparison with Lusztig's equivalent definition of a Drinfeld (graded) Hecke algebra in which the group action relations are deformed. 
Proof. Assume $\mathscr{H}_{\kappa, t}$ is a Drinfeld orbifold algebra over $k[t]$. Let $v_{1}, \ldots, v_{n}$ be a basis of the vector space $V$, so that

$$
\left\{v_{1}^{i_{1}} \cdots v_{n}^{i_{n}} \mid i_{1}, \ldots, i_{n} \in \mathbb{Z}_{\geq 0}\right\}
$$

is a basis of $S$. Since gr $\mathscr{H}_{\kappa} \cong S \# G$, there is a corresponding basis $\mathscr{B}$ of $\mathscr{H}_{\kappa}$ given by all $v_{1}^{i_{1}} \cdots v_{n}^{i_{n}} g$, where $g$ ranges over all elements in $G$ and $i_{1}, \ldots, i_{n}$ range over all nonnegative integers. Hence, we may identify $\mathscr{H}_{\kappa, t}$ with $S \# G[t]$ as a $k$-vector space. As $\mathscr{H}_{\kappa, t}$ is associative, it defines a deformation of $S \# G[t]$ as follows.

Let $r=v_{1}^{i_{1}} \cdots v_{n}^{i_{n}} g$ and $s=v_{1}^{j_{1}} \cdots v_{n}^{j_{n}} h$ be elements of $\mathscr{B}$. For clarity, we denote the product in $\mathscr{H}_{\kappa, t}$ by $*$. Using the relations of $\mathscr{H}_{\kappa, t}$ to express the product $r * s$ as a linear combination of elements of $\mathscr{B}$, we may expand uniquely:

$$
r * s=r s+\mu_{1}(r, s) t+\mu_{2}(r, s) t^{2}+\cdots+\mu_{m}(r, s) t^{m}
$$

for some $m=m_{r, s}$ depending on $r, s$, and some $\mu_{1}, \ldots, \mu_{m}$. By the definition of $\mathscr{H}_{\kappa, t}$ as a quotient of $T(V) \# G[t]$, the group algebra $k G$ is in the kernel of $\mu_{i}$ for all $i$. Using the relations in $\mathscr{H}_{\kappa}$, we have

$$
r * s=\left(\left(v_{1}^{i_{1}} \cdots v_{n}^{i_{n}}\right) *\left({ }^{g}\left(v_{1}^{j_{1}} \cdots v_{n}^{j_{n}}\right)\right)\right) g h .
$$

We apply the relations of $\mathscr{H}_{\kappa, t}$ repeatedly to rewrite the product $\left(v_{1}^{i_{1}} \cdots v_{n}^{i_{n}}\right) *$ $\left({ }^{g}\left(v_{1}^{j_{1}} \cdots v_{n}^{j_{n}}\right)\right)$ as an element in the $k$-span of $\mathscr{B}$. We prove by induction on the degree $d=\sum_{l=1}^{n}\left(i_{l}+j_{l}\right)$ that $\operatorname{deg} \mu_{i}=-i$. It suffices to prove this in case $g=1$. If $d=0$ or $d=1$, the maps $\mu_{i}$ give 0 , and so they satisfy the degree requirement trivially. Similarly, whenever $a<b, v_{a} * v_{b}$ in $\mathscr{H}_{\kappa, t}$ identifies with $v_{a} v_{b}$ in $S$, and $\mu_{i}\left(v_{a}, v_{b}\right)=0$ for all $i$. Thus if $d=2$, the nontrivial case is when some $i_{l}=1$ and some $j_{m}=1$ with $l>m$. Then

$$
v_{l} * v_{m}-v_{m} * v_{l}=\kappa^{L}\left(v_{l}, v_{m}\right) t+\kappa^{C}\left(v_{l}, v_{m}\right) t^{2} .
$$

By construction, $\mu_{1}\left(v_{l}, v_{m}\right)=\kappa^{L}\left(v_{l}, v_{m}\right)$, an element of $V \otimes k G$, and the map $\mu_{1}$ has degree -1 on this input. Similarly, $\mu_{2}\left(v_{l}, v_{m}\right)=\kappa^{C}\left(v_{l}, v_{m}\right)$, which has degree -2 on this input.

Now assume $d>2$ is arbitrary and $d=\sum_{l=1}^{n}\left(i_{l}+j_{l}\right)$. Without loss of generality, assume $i_{n} \geq 1, j_{1} \geq 1$, and then

$$
\begin{aligned}
\left(v_{1}^{i_{1}} \cdots v_{n}^{i_{n}}\right) *\left(v_{1}^{j_{1}} \cdots v_{n}^{j_{n}}\right)=\left(v_{1}^{i_{1}} \cdots\right. & \left.v_{n}^{i_{n}-1}\right) *\left(v_{1} v_{n} * v_{1}^{j_{1}-1} \cdots v_{n}^{j_{n}}\right) \\
& +\left(v_{1}^{i_{1}} \cdots v_{n}^{i_{n}-1}\right) *\left(\kappa^{L}\left(v_{n}, v_{1}\right) * v_{1}^{j_{1}-1} \cdots v_{n}^{j_{n}}\right) t \\
& +\left(v_{1}^{i_{1}} \cdots v_{n}^{i_{n}-1}\right) *\left(\kappa^{C}\left(v_{n}, v_{1}\right) * v_{1}^{j_{1}-1} \cdots v_{n}^{j_{n}}\right) t^{2} .
\end{aligned}
$$

In the second and third terms, we see that the degree lost by applying the map is precisely that gained in the power of $t$. In the first term, no degree was lost and no power of $t$ was gained, however the factors are one step closer to being part of a 
PBW basis. By induction, the degrees of the $\mu_{i}$ are as claimed. Equivalently, we may give $t$ a degree of 1 , making $\mathscr{H}_{\kappa, t}$ a graded algebra, and argue as in [Braverman and Gaitsgory 1996; Du et al. 2007].

Now assume that $A$ is any deformation of $S \# G$ over $k[t]$ for which $\operatorname{deg} \mu_{i}=$ $-i$ and for which $k G$ is in the kernel of $\mu_{i}$ for all $i \geq 1$. By definition, $A$ is isomorphic to $S \# G[t]$ as a vector space over $k[t]$. Fix a basis $v_{1}, \ldots, v_{n}$ of $V$. Let $\phi: T(V) \# G[t] \rightarrow A$ be the $k[t]$-linear map given by

$$
\phi\left(v_{i_{1}} \cdots v_{i_{m}} g\right)=v_{i_{1}} * \cdots * v_{i_{m}} * g
$$

for all words $v_{i_{1}} \cdots v_{i_{m}}$ and group elements $g$. Since $T(V)$ is free on $v_{1}, \ldots, v_{n}$ and by hypothesis, $\mu_{i}(k G, k G)=\mu_{i}(k G, V)=\mu_{i}(V, k G)=0$ for all $i \geq 1$, the map $\phi$ is in fact an algebra homomorphism. It may be shown by induction on degree that $\phi$ is surjective, using the degree hypothesis on the maps $\mu_{i}$.

We next find the kernel of $\phi$. Let $v, w \in V$ be elements of the basis. Then

$$
\begin{aligned}
& \phi(v w)=v * w=v w+\mu_{1}(v, w) t+\mu_{2}(v, w) t^{2}, \\
& \phi(w v)=w * v=w v+\mu_{1}(w, v) t+\mu_{2}(w, v) t^{2},
\end{aligned}
$$

since $\operatorname{deg} \mu_{i}=-i$ for each $i$. Since $v w=w v$ in $S$, we have

$$
\phi(v w-w v)=\left(\mu_{1}(v, w)-\mu_{1}(w, v)\right) t-\left(\mu_{2}(v, w)-\mu_{2}(w, v)\right) t^{2} .
$$

It follows that

$$
v w-w v-\left(\mu_{1}(v, w)-\mu_{1}(w, v)\right) t-\left(\mu_{2}(v, w)-\mu_{2}(w, v)\right) t^{2}
$$

is in the kernel of $\phi$, since $\phi(v g)=v g$ and $\phi(g)=g$ for all $v \in V$ and $g \in G$. By the degree conditions on the $\mu_{i}$, there are functions $\kappa_{g}^{L}: V \wedge V \rightarrow V \otimes k G$ and $\kappa_{g}^{C}: V \wedge V \rightarrow k G$ for all $g \in G$ such that

$$
\begin{aligned}
& \mu_{1}(v, w)-\mu_{1}(w, v)=\sum_{g \in G} \kappa_{g}^{L}(v, w) g, \\
& \mu_{2}(v, w)-\mu_{2}(w, v)=\sum_{g \in G} \kappa_{g}^{C}(v, w) g .
\end{aligned}
$$

For each $g \in G$, the functions $\kappa_{g}^{C}: V \wedge V \rightarrow k G$ and $\kappa_{g}^{L}: V \wedge V \rightarrow V \otimes k G$ are linear (by their definitions). Let $I[t]$ be the ideal of $T(V) \# G[t]$ generated by all expressions of the form (2.2), so by definition $I[t] \subset \operatorname{Ker} \phi$. We claim that in fact $I[t]=\operatorname{Ker} \phi$ : The quotient $T(V) \# G[t] / I[t]$ is by definition a filtered algebra over $k[t]$ whose associated graded algebra is necessarily $S \# G[t]$ or a quotient thereof. By a dimension count in each degree, since $I[t] \subset \operatorname{Ker} \phi$, this forces $I[t]=\operatorname{Ker} \phi$. Therefore $\phi$ induces an isomorphism from $\mathscr{H}_{\kappa, t}$ to $A$ and thus the deformation $A$ of $S \# G$ is isomorphic to a Drinfeld orbifold algebra. 
Remark 2.5. When working with a Drinfeld orbifold algebra, we may always assume the relations (2.3) and (2.4) hold for $v, w$ in $V$ as a consequence of the proof. In a later section, we will make more explicit this connection between the functions $\mu_{i}$ and $\kappa$, using Hochschild cohomology in case the characteristic of $k$ does not divide the order of $G$ : We will consider the $\mu_{i}$ to be cochains on the bar resolution of $S \# G$, and $\kappa$ to be a cochain on the Koszul resolution of $S$. The relations (2.3) and (2.4) then result from applying chain maps to convert between the two resolutions. Specifically, let $\phi$. be a map from the Koszul resolution to the bar resolution of $S$ (a subcomplex of the bar resolution of $S \# G$ ). Then $\kappa^{L}=\mu_{1} \circ \phi_{2}$, as we will explain.

\section{Necessary and sufficient conditions}

We determine conditions on the parameter $\kappa$ for $\mathscr{H}=\mathscr{H}_{\kappa}$ to satisfy the PBW condition. In the setting of symplectic reflection algebras over the complex numbers, Etingof and Ginzburg [2002, Theorem 1.3] used a generalization of [Braverman and Gaitsgory 1996, Theorem 0.5 and Lemma 3.3] that replaces the ground field $k$ with the (semisimple) group ring $k G$. This approach was then adopted in [Halbout et al. 2011]. Since the generalization of the work of Braverman and Gaitsgory does not immediately apply in arbitrary characteristic, and since one of the conditions in [Halbout et al. 2011] is missing a factor of 2, we include two proofs of the PBW conditions for Drinfeld orbifold algebras, one using a Braverman-Gaitsgory approach and one using Bergman's [1978] diamond lemma. The second proof applies in all characteristics other than 2, even those dividing the order of $G$, while the first requires $k G$ to be semisimple. (See [Khare 2007] for the diamond lemma argument applied in a related setting and see [Levandovskyy and Shepler 2011] for a related approach using noncommutative Gröbner theory, but in a quantum setting.)

The set of all parameter functions

$$
\kappa: V \wedge V \rightarrow(k \oplus V) \otimes k G
$$

(defining the quotient algebras $\left.\mathscr{H}_{\kappa}\right)$ carries the usual induced $G$-action: $\left({ }^{h} \kappa\right)(*)=$ ${ }^{h}\left(\kappa\left(h^{-1}(*)\right)\right)$, i.e., for all $h \in G$ and $v, w \in V$,

$$
\left({ }^{h} \kappa\right)(v, w)={ }^{h}\left(\kappa\left(h^{-1} v, h^{-1} w\right)\right)=\sum_{g \in G}{ }^{h}\left(\kappa_{g}\left(\left(^{-1} v,{ }^{-1} w\right)\right) h g h^{-1} .\right.
$$

We say that $\kappa$ is $G$-invariant when ${ }^{h} \kappa=\kappa$ for all $h$ in $G$. Let $\mathrm{Alt}_{3}$ denote the cyclic group of order 3 considered as a subgroup of the symmetric group on 3 symbols. Note that the following theorem gives conditions in the symmetric algebra $S$. We allow the field $k$ to have arbitrary characteristic other than 2 . 
Theorem 3.1. The algebra $\mathscr{H}_{\kappa}$ is a Drinfeld orbifold algebra if and only if the following conditions hold for each $g$ in $G$ and $v_{1}, v_{2}, v_{3}$ in $V$ :

(i) The parameter function $\kappa$ is $G$-invariant.

(ii)

$$
\sum_{\sigma \in \mathrm{Alt}_{3}} \kappa_{g}^{L}\left(v_{\sigma(2)}, v_{\sigma(3)}\right)\left(v_{\sigma(1)}-{ }^{g} v_{\sigma(1)}\right)=0 \text { in } S=S(V) .
$$

$$
\begin{aligned}
\sum_{\sigma \in \mathrm{Alt}_{3}} \sum_{h \in G} \kappa_{g h^{-1}}^{L}\left(v_{\sigma(1)}+{ }^{h} v_{\sigma(1)},\right. & \left.\kappa_{h}^{L}\left(v_{\sigma(2)}, v_{\sigma(3)}\right)\right) \\
& =2 \sum_{\sigma \in \mathrm{Alt}_{3}} \kappa_{g}^{C}\left(v_{\sigma(2)}, v_{\sigma(3)}\right)\left({ }^{g} v_{\sigma(1)}-v_{\sigma(1)}\right) .
\end{aligned}
$$

(iv) $\sum_{\sigma \in \mathrm{Alt}_{3}} \sum_{h \in G} \kappa_{g h^{-1}}^{C}\left(v_{\sigma(1)}+{ }^{h} v_{\sigma(1)}, \kappa_{h}^{L}\left(v_{\sigma(2)}, v_{\sigma(3)}\right)\right)=0$.

Proof of Theorem 3.1 using the theory of Koszul rings over $k G$. In this proof, we restrict to the case where the characteristic of $k$ does not divide the order of $G$. The skew group algebra $S \# G$ is then a Koszul ring over $k G$, as defined by Beilinson, Ginzburg, and Soergel (see [Beilinson et al. 1996, Definition 1.1.2 and Section 2.6]). These authors worked with graded algebras in which the degree 0 component is not necessarily commutative, but is a semisimple algebra. In our case the degree 0 component of $S \# G$ is the semisimple group algebra $k G$. The results of Braverman and Gaitsgory [1996, Theorem 0.5 and Lemma 3.3] can be extended to this general setting to give necessary and sufficient conditions on $\kappa$ under which $\mathscr{H}_{\kappa}$ is a Drinfeld orbifold algebra (cf. [Etingof and Ginzburg 2002, proof of Theorem 1.3]).

We first write $T_{k}(V) \# G$ as a tensor algebra over $k G$. We give the underlying vector space $T_{k}(V) \otimes k G$ a $k G$-bimodule structure by setting $g(v \otimes h):={ }^{g} v \otimes g h$ and $(v \otimes h) g:=v \otimes h g$ for all $v$ in $T_{k}(V)$ and all $g, h$ in $G$. Let $T$ be the tensor algebra of $V \otimes k G$ over $k G$. Then the $k G$-bimodule structure on $V \otimes k G$ (restricted from that on $T_{k}(V) \otimes k G$ ) extends to a $k G$-bimodule structure on $T$. We use the abbreviation $v g$ for each element $v \otimes g$ in $V \otimes k G$ and identify $V$ with $V \otimes 1_{G}$ in $V \otimes k G$. Then $T:=T_{k G}(V \otimes k G)$ is isomorphic to $T_{k}(V) \# G$ as an algebra and as a $k G$-module via the map sending any $v_{1} \otimes_{k G} \cdots \otimes_{k G}\left(v_{m} g\right)$ in $T$ to $\left(v_{1} \otimes \cdots \otimes v_{m}\right) \otimes g$ in $T_{k}(V) \# G$. For ease with notation (and to avoid confusion with tensor signs), we identify these spaces. Thus, we may write the standard filtration $F$ on $T$ as

$$
\begin{aligned}
& F^{0}(T)=k G, \\
& F^{1}(T)=k G \oplus(V \otimes k G), \\
& F^{2}(T)=k G \oplus(V \otimes k G) \oplus(V \otimes V \otimes k G),
\end{aligned}
$$

and so on. 
Now let $P$ be the $k G$-subbimodule of $T$ generated by all $v \otimes w-w \otimes v-\kappa(v, w)$, for $v, w \in V$. Let $R$ be the $k G$-subbimodule generated by all $v \otimes w-w \otimes v$, for $v, w \in V$. By [Braverman and Gaitsgory 1996, Theorem 0.5], $\mathscr{H}_{\kappa} \cong T_{k G}(V \otimes k G) / P$ is a Drinfeld orbifold algebra if and only if

(I) $P \cap F^{1}(T)=0$ and

(J) $\left(F^{1}(T) \cdot P \cdot F^{1}(T)\right) \cap F^{2}(T)=P$.

By [ibid., Lemma 3.3], if (I) holds, then (J) is equivalent to the following three conditions, where $\alpha: R \rightarrow V \otimes k G, \beta: R \rightarrow k G$ are maps for which

$$
P=\{r-\alpha(r)-\beta(r) \mid r \in R\}:
$$

(a) $\operatorname{Im}(\alpha \otimes \mathrm{id}-\mathrm{id} \otimes \alpha) \subset R$.

(b) $\alpha \circ(\alpha \otimes \mathrm{id}-\mathrm{id} \otimes \alpha)=-(\beta \otimes \mathrm{id}-\mathrm{id} \otimes \beta)$.

(c) $\beta \circ(\mathrm{id} \otimes \alpha-\alpha \otimes \mathrm{id}) \equiv 0$.

The maps $\alpha \otimes \mathrm{id}-\mathrm{id} \otimes \alpha$ and $\beta \otimes \mathrm{id}-\mathrm{id} \otimes \beta$ above are defined on the intersection $\left(R \otimes_{k G}(V \otimes k G)\right) \cap\left((V \otimes k G) \otimes_{k G} R\right)$. Extend $\kappa$ to an alternating $k G$-module map on $T^{2}:=T_{k G}^{2}(V \otimes k G)$ so that $\kappa(g(v \otimes w) h)=\kappa\left({ }^{g} v \otimes{ }^{g} w\right) g h$ for all $g, h$ in $G$ and $v, w$ in $V$. (Note that this is the only possible way to extend $\kappa$ if $\kappa$ is invariant.) Then $\alpha(v \otimes w-w \otimes v)=\kappa^{L}(v \otimes w)=\frac{1}{2} \kappa^{L}(v \otimes w-w \otimes v)$ (as $\kappa$ is alternating) and for all $r$ in $R$,

$$
2 \alpha(r)=\kappa^{L}(r) .
$$

(Similarly, $2 \beta(r)=\kappa^{C}(r)$ for all $r$ in $R$.)

First note that (I) is equivalent to the condition that $G$ preserves the vector space generated by all $v \otimes w-w \otimes v-\kappa^{L}(v, w)-\kappa^{C}(v, w)$, i.e., this space contains

$$
{ }^{g} v \otimes{ }^{g} w-{ }^{g} w \otimes{ }^{g} v-{ }^{g}\left(\kappa^{L}(v, w)\right)-{ }^{g}\left(\kappa^{C}(v, w)\right)
$$

for each $g \in G, v, w \in V$. Equivalently, we have

$$
\begin{aligned}
& \kappa^{L}\left({ }^{g} v,{ }^{g} w\right)={ }^{g}\left(\kappa^{L}(v, w)\right), \\
& \kappa^{C}\left({ }^{g} v,{ }^{g} w\right)={ }^{g}\left(\kappa^{C}(v, w)\right) ;
\end{aligned}
$$

that is, both $\kappa^{L}$ and $\kappa^{C}$ are $G$-invariant, yielding (i).

We assume now that $\kappa$ is $G$-invariant and proceed with the remaining conditions.

Condition (a): As a $k G$-bimodule, $\left(R \otimes_{k G}(V \otimes k G)\right) \cap\left((V \otimes k G) \otimes_{k G} R\right)$ is generated by elements of the form $\sum_{\sigma \in S_{3}}(\operatorname{sgn} \sigma) v_{\sigma(1)} \otimes v_{\sigma(2)} \otimes v_{\sigma(3)}$, so we find the image of $\alpha \otimes \mathrm{id}-\mathrm{id} \otimes \alpha$ on these elements. After reindexing, we obtain

$$
\sum_{\sigma \in \mathrm{Alt}_{3}}\left(\kappa^{L}\left(v_{\sigma(2)}, v_{\sigma(3)}\right) \otimes v_{\sigma(1)}-v_{\sigma(1)} \otimes \kappa^{L}\left(v_{\sigma(2)}, v_{\sigma(3)}\right)\right) .
$$


We decompose into components indexed by $g$ in $G$ and shift all group elements to the right (tensor products are over $k G$ ). The $g$-th summand is then

$$
\sum_{\sigma \in \mathrm{Alt}_{3}}\left(\kappa_{g}^{L}\left(v_{\sigma(2)}, v_{\sigma(3)}\right) \otimes{ }^{g} v_{\sigma(1)}-v_{\sigma(1)} \otimes \kappa_{g}^{L}\left(v_{\sigma(2)}, v_{\sigma(3)}\right)\right) g,
$$

which must be an element of $R$. This is equivalent to the vanishing of its image in $S \# G$. We rewrite this as (ii).

Condition (b): We assume condition (a) holds and thus (3.2) is an element of $R$. We compute the left side of condition (b) by applying $\alpha$ to this element. Since $2 \alpha(r)=\kappa^{L}(r)$ for all $r$ in $R$, we obtain the left side of (iii) after dividing by 2. Similarly, it is not difficult to see that the right side of condition (b) agrees with the right side of (iii): The image of $\sum_{\sigma \in S_{3}}(\operatorname{sgn} \sigma) v_{\sigma(1)} \otimes v_{\sigma(2)} \otimes v_{\sigma(3)}$ under $-(\beta \otimes \mathrm{id}-\mathrm{id} \otimes \beta)$ is

$$
-\sum_{\substack{\sigma \in \mathrm{Alt}_{3} \\ g \in G}}\left(\kappa_{g}^{C}\left(v_{\sigma(2)}, v_{\sigma(3)}\right) \otimes{ }^{g} v_{\sigma(1)}-v_{\sigma(1)} \otimes \kappa_{g}^{C}\left(v_{\sigma(2)}, v_{\sigma(3)}\right)\right) g
$$

(as an element of $V \otimes k G$ ) which we rewrite as

$$
-\sum_{\substack{\sigma \in \mathrm{Alt}_{3} \\ g \in G}}\left(\kappa_{g}^{C}\left(v_{\sigma(2)}, v_{\sigma(3)}\right)\left({ }^{g} v_{\sigma(1)}-v_{\sigma(1)}\right)\right) g .
$$

Condition (c): An analysis similar to that for condition (b) yields (iv).

Proof of Theorem 3.1 using the diamond lemma. In this proof, the characteristic of $k$ may be 0 or any odd prime. We apply [Bergman 1978] to obtain conditions on $\kappa$ equivalent to existence of a PBW basis and then argue that these conditions are equivalent to those in the theorem. We suppress details and merely record highlights of the argument (which requires one to fix a monomial ordering and check all overlap/inclusion ambiguities on the set of relations defining $\mathscr{H}_{\kappa}$ ), as described, for example, in [Bueso et al. 2003, Chapter 3]). Fix a basis $v_{1}, \ldots, v_{n}$ of $V$ and let $\mathscr{B}$ be our prospective PBW basis: Set $\mathscr{B}=\left\{v_{1}^{\alpha_{1}} \cdots v_{n}^{\alpha_{n}} g: \alpha_{i} \in \mathbb{Z}_{\geq 0}, g \in G\right\} \subset T(V) \otimes k G$, a subset of the free algebra $\mathscr{F}$ generated by $v$ in $V$ and $g$ in $G$.

Using the diamond lemma, one may show that necessary and sufficient conditions for $\mathscr{H}_{\kappa}$ to satisfy the PBW condition arise from expanding conjugation and Jacobi identities in $\mathscr{H}_{\kappa}$ : For every choice of parameter $\kappa$, and for every $v, w$ in $V$ and $h$ in $G$, the elements

$$
h[v, w] \mathscr{H} h^{-1}-\left[{ }^{h} v,{ }^{h} w\right] \mathscr{H}
$$

and

$$
\left[v_{i},\left[v_{j}, v_{\ell}\right]_{\mathscr{H}}\right]_{\mathscr{H}}+\left[v_{j},\left[v_{\ell}, v_{i}\right]_{\mathscr{H}}\right]_{\mathscr{H}}+\left[v_{\ell},\left[v_{i}, v_{j}\right]_{\mathscr{H}}\right]_{\mathscr{H}}
$$


are always zero in the associative algebra $\mathscr{H}$. Here, $[a, b] \mathscr{H}:=a b-b a$ is just the commutator in $\mathscr{H}$ of $a, b \in \mathscr{H}$. Using the relations defining $\mathscr{H}$, we move all group elements to the right and arrange indices of basis vectors in increasing order (apply straightening operations).

An analysis of elements of type (1) shows that a PBW property on $\mathscr{H}_{\kappa}$ forces ${ }^{h} \kappa=\kappa$ for all $h$ in $G$. Indeed, this condition is equivalent to

$$
\kappa_{h^{-1} g h}(v, w)={ }^{h^{-1}}\left(\kappa_{g}\left({ }^{h} v,{ }^{h} w\right)\right) \quad \text { for all } g, h \in G, v, w \in V .
$$

We next write each element of type (2) above in the image under the projection map $\pi: \mathscr{F} \rightarrow \mathscr{H}$ of some $f\left(v_{i}, v_{j}, v_{\ell}\right)$ in the $k$-span of (potentially) nonzero elements of $\mathscr{B}$. Take (for example) the index set $\{i, j, \ell\}=\{1,2,3\}$. In $\mathscr{H}_{\kappa}$,

$$
\begin{aligned}
{\left[v_{1},\left[v_{2}, v_{3}\right]_{\mathscr{H}}\right]_{\mathscr{H}}=v_{1} \kappa\left(v_{2}, v_{3}\right)-\kappa\left(v_{2}, v_{3}\right) v_{1} } \\
\quad=\sum_{g \in G} v_{1} \kappa_{g}^{C}\left(v_{2}, v_{3}\right) g-\kappa_{g}^{C}\left(v_{2}, v_{3}\right) g v_{1}+v_{1} \kappa_{g}^{L}\left(v_{2}, v_{3}\right) g-\kappa_{g}^{L}\left(v_{2}, v_{3}\right) g v_{1} \\
\quad=\sum_{g \in G}\left(v_{1} \kappa_{g}^{C}\left(v_{2}, v_{3}\right)-\kappa_{g}^{C}\left(v_{2}, v_{3}\right)^{g} v_{1}+v_{1} \kappa_{g}^{L}\left(v_{2}, v_{3}\right)-\kappa_{g}^{L}\left(v_{2}, v_{3}\right)^{g} v_{1}\right) g \\
\quad=\sum_{g \in G}\left(\kappa_{g}^{C}\left(v_{2}, v_{3}\right)\left(v_{1}-{ }^{g} v_{1}\right)+v_{1} \kappa_{g}^{L}\left(v_{2}, v_{3}\right)-\kappa_{g}^{L}\left(v_{2}, v_{3}\right)^{g} v_{1}\right) g .
\end{aligned}
$$

We apply further relations in $\mathscr{H}$ to this last expression to rearrange the vectors $v_{1}, \ldots, v_{n}$ by adding terms of lower degree. Thus, if we express $f=f\left(v_{1}, v_{2}, v_{3}\right)$ as $f_{0}+f_{1}+f_{2}$ where $f_{i}$ has degree $i$ in the free algebra $\mathscr{F}_{F}$, then $\pi\left(f_{2}\right)$ and

$$
\sum_{g \in G} \sum_{\sigma \in \mathrm{Alt}_{3}} \kappa_{g}^{L}\left(v_{\sigma(2)}, v_{\sigma(3)}\right)\left(v_{\sigma(1)}-{ }^{g} v_{\sigma(1)}\right) g
$$

differ only by a rearrangement of vectors: They both project to the same element under $T(V) \otimes k G \rightarrow S(V) \otimes k G$. But $f_{2}$ is zero in the free algebra $\mathscr{F}$ if and only if its image is zero in $S(V) \otimes k G$, yielding (ii).

The other conditions of the theorem require a bit of manipulation. One may show that

$$
\begin{aligned}
f=\sum_{\substack{\sigma \in \mathrm{Alt}_{3} \\
g \in G}}\left(\kappa _ { g } ^ { C } ( v _ { \sigma ( 2 ) } , v _ { \sigma ( 3 ) } ) \left(v_{\sigma(1)}-\right.\right. & \left.{ }^{g} v_{\sigma(1)}\right) \\
& \left.+\sum_{a<b}\left(D_{a b}^{g}+D_{b a}^{g}\right) v_{a} v_{b}-D_{a b}^{g} \kappa\left(v_{a}, v_{b}\right)\right) g,
\end{aligned}
$$

where the $D_{a b}^{g}$ in $k$ are constants determined by the action of $G$ on $V$ and the values of $\kappa$ expanded in terms of the fixed basis of $V$. Specifically, $D_{a b}^{g}:=$ $\delta_{b, \sigma(1)} c_{a}^{\sigma(2), \sigma(3), g}-c_{b}^{\sigma(2), \sigma(3), g} d_{a}^{\sigma(1), g}$ where $\delta_{a, b}$ is the Kronecker delta symbol and where ${ }^{g} v_{a}=\sum_{b} d_{b}^{a, g} v_{b}$ and $\kappa_{g}^{L}\left(v_{a}, v_{b}\right)=\sum_{m} c_{m}^{a, b, g} v_{m}$. 
Note that $f_{2}$ is zero if and only if $\sum_{\sigma \in \mathrm{Alt}_{3}}\left(D_{a b}^{g}+D_{b a}^{g}\right)=0$ for all $a<b$ and $g$ in $G$. Thus, whenever $f_{2}$ is zero, we may substitute $D_{a b}^{g}=-D_{b a}^{g}$ in the equation $0=2\left(f_{0}+f_{1}\right)$ to see that $f_{0}+f_{1}$ vanishes exactly when

$$
2 \sum_{g \in G} \kappa^{C}\left(v_{\sigma(2)}, v_{\sigma(3)}\right)\left(v_{\sigma(1)}-{ }^{g} v_{\sigma(1)}\right) g=\sum_{\substack{\sigma \in \mathrm{Alt}_{3} \\ g \in G}} \sum_{a<b}\left(D_{a b}^{g}-D_{b a}^{g}\right) \kappa\left(v_{a}, v_{b}\right) g .
$$

We write the right-hand side as a sum over all $a$ and $b$ (as $\kappa$ is alternating) and obtain

$$
\sum_{\substack{\sigma \in \mathrm{Alt}_{3} \\ g \in G}} \kappa\left(\kappa_{g}^{L}\left(v_{\sigma(2)}, v_{\sigma(3)}\right), v_{\sigma(1)}+{ }^{g} v_{\sigma(1)}\right) g .
$$

This yields (iii) and (iv) of the theorem whenever (ii) holds.

Thus, the four conditions of the theorem are equivalent to $G$-invariance of $\kappa$ and the vanishing of all $f_{0}, f_{1}, f_{2}$ (for any $i, j, \ell$ ), which in turn is equivalent to the PBW property for $\mathscr{H}_{\kappa}$ by careful application of the diamond lemma.

We illustrate the theorem by giving two examples for which $\kappa^{C}$ is identically 0 . In the next section we give an example for which $\kappa^{L}$ and $\kappa^{C}$ are both nonzero.

Example 3.3. Let $G \cong \mathbb{Z} / 2 \mathbb{Z} \times \mathbb{Z} / 2 \mathbb{Z}$, with generators $g$ and $h$, act on the complex vector space $V$ having basis $x, y, z$ by:

$$
\begin{array}{lll}
{ }^{g} x=-x, & { }^{g} y=y, & { }^{g} z=-z, \\
{ }^{h} x=-x, & { }^{h} y=-y, & { }^{h} z=z .
\end{array}
$$

Define an alternating bilinear map $\kappa^{L}: V \times V \rightarrow V \otimes k G$ by

$$
\kappa^{L}(x, y)=z h, \quad \kappa^{L}(y, z)=x g h, \quad \kappa^{L}(z, x)=y g,
$$

and let $\kappa^{C} \equiv 0$. One checks that $\kappa^{L}$ is $G$-invariant and that conditions (ii) and (iii) of Theorem 3.1 hold. Condition (iv) holds automatically since $\kappa^{C}$ is identically 0 . The corresponding Drinfeld orbifold algebra is

$$
T(V) \# G /([x, y]-z h,[y, z]-x g h,[z, x]-y g) .
$$

Example 3.4. Let $G=S_{3}$ act by permutations on a basis $v_{1}, v_{2}, v_{3}$ of a complex three-dimensional vector space $V$. Let $\xi$ be a primitive cube root of 1 , and let

$$
w_{1}=v_{1}+\xi v_{2}+\xi^{2} v_{3}, \quad w_{2}=v_{1}+\xi^{2} v_{2}+\xi v_{3}, \quad w_{3}=v_{1}+v_{2}+v_{3} .
$$

Define an alternating bilinear map $\kappa^{L}: V \times V \rightarrow V \otimes k G$ by

$$
\kappa^{L}\left(w_{1}, w_{2}\right)=w_{3}((1,2,3)-(1,3,2)), \quad \kappa^{L}\left(w_{2}, w_{3}\right)=0, \quad \kappa^{L}\left(w_{1}, w_{3}\right)=0,
$$

and let $\kappa^{C} \equiv 0$, where $(1,2,3),(1,3,2)$ are the standard 3-cycles in $S_{3}$. One checks that $\kappa^{L}$ is $G$-invariant and that conditions (ii) and (iii) of Theorem 3.1 hold. 
Condition (iv) holds automatically since $\kappa^{C}$ is identically zero. The corresponding Drinfeld orbifold algebra is

$$
T(V) \# G /\left(\left[w_{1}, w_{2}\right]-w_{3}((1,2,3)-(1,3,2)), \quad\left[w_{2}, w_{3}\right], \quad\left[w_{1}, w_{3}\right]\right) .
$$

The conditions of Theorem 3.1 simplify significantly when $\kappa^{L}$ is supported on the identity element $1:=1_{G}$ of $G$ alone, and we turn to this interesting case in the next section.

\section{Lie orbifold algebras}

The universal enveloping algebra of a finite-dimensional Lie algebra is a special case of a Drinfeld orbifold algebra. We extend universal enveloping algebras by groups and explore deformations of the resulting algebras in this section. Assume throughout this section that the linear part of our parameter $\kappa$ is supported on the identity $1=1_{G}$ of $G$ alone, that is, $\kappa_{g}^{L} \equiv 0$ for all $g \in G-\{1\}$. It is convenient in this section to use standard notation from the theory of Lie algebras and Drinfeld Hecke algebras (i.e., graded Hecke algebras): Let

$$
a_{g}: V \wedge V \rightarrow k \quad(\text { for all } g \text { in } G)
$$

and

$$
[\cdot, \cdot]_{\mathfrak{g}}: V \wedge V \rightarrow V
$$

be linear functions where $\mathfrak{g}:=V$ as a vector space with the additional structure given by the map $[\cdot, \cdot]_{\mathfrak{g}}$. Define an algebra $\mathscr{H}:=\mathscr{H}\left(\mathfrak{g} ; a_{g}, g \in G\right)$ as the quotient

$$
\mathscr{H}=T(V) \# G /\left(v w-w v-[v, w]_{\mathfrak{g}}-\sum_{g \in G} a_{g}(v, w) g \mid v, w \in V\right) .
$$

Then $\mathscr{H}$ is a filtered algebra by its definition. We say that $\mathscr{H}$ is a Lie orbifold algebra when it satisfies the PBW condition, that is, when $\operatorname{gr} \mathscr{H} \cong S \# G$. We determine necessary and sufficient conditions on the functions $[\cdot, \cdot]_{\mathfrak{g}}$ and $a_{g}$ for $\mathscr{H}$ to be a Lie orbifold algebra. We will see that the PBW condition implies that the bracket $[\cdot, \cdot]_{\mathfrak{g}}$ endows $\mathfrak{g}=V$ with the structure of a Lie algebra (carrying an action of $G$ by automorphisms), thus explaining the choice of notation and terminology.

Proposition 4.1. The quotient

$$
\mathscr{H}=T(V) \# G /\left(v \otimes w-w \otimes v-[v, w]_{\mathfrak{g}}-\sum_{g \in G} a_{g}(v, w) g \mid v, w \in V\right)
$$

defines a Lie orbifold algebra if and only if three conditions hold:

1. The bracket $[\cdot, \cdot]_{\mathfrak{g}}$ is a $G$-invariant Lie bracket (and thus $\mathfrak{g}:=V$ is a Lie algebra upon which $G$ acts by automorphisms). 
2. The parameters $\left\{a_{g}\right\}_{g \in G}$ define a Drinfeld Hecke algebra, that is,

$$
a_{h g h^{-1}}(v, w)=a_{g}\left({ }^{h} v,{ }^{h} w\right) \quad \text { for all } v, w \in V \text { and } g, h \in G,
$$

and the Jacobi identity holds: for all $v_{i} \in V$ and $g \in G$,

$$
0=a_{g}\left(v_{2}, v_{3}\right)\left(v_{1}-{ }^{g} v_{1}\right)+a_{g}\left(v_{3}, v_{1}\right)\left(v_{2}-{ }^{g} v_{2}\right)+a_{g}\left(v_{1}, v_{2}\right)\left(v_{3}-{ }^{g} v_{3}\right) .
$$

3. The Lie bracket and Drinfeld Hecke algebra structures are compatible:

$$
0=a_{g}\left(v_{3},\left[v_{1}, v_{2}\right]_{\mathfrak{g}}\right)+a_{g}\left(v_{1},\left[v_{2}, v_{3}\right]_{\mathfrak{g}}\right)+a_{g}\left(v_{2},\left[v_{3}, v_{1}\right]_{\mathfrak{g}}\right)
$$

for all $v_{i} \in V$ and $g \in G$.

Proof. Theorem 3.1(i) is equivalent to $G$-invariance of the Lie bracket $[\cdot, \cdot]_{\mathfrak{g}}$ and the first equation in condition 2.

We next examine conditions (ii), (iii), and (iv) of Theorem 3.1, resulting from the Jacobi identity on $\mathscr{H}$,

$$
0=\left[v_{1},\left[v_{2}, v_{3}\right]_{\mathscr{H}}\right] \mathscr{H}+\left[v_{2},\left[v_{3}, v_{1}\right]_{\mathscr{H}}\right]_{\mathscr{H}}+\left[v_{3},\left[v_{1}, v_{2}\right]_{\mathscr{H}}\right] \mathscr{H} \text { for all } v_{i} \in V,
$$

after setting $\kappa^{L}(v, w)=[v, w]_{\mathfrak{g}}$ and $\kappa^{C}(v, w)=\sum_{g \in G} a_{g}(v, w) g$. When $g=1$, (ii) holds automatically, and (iii) is equivalent to the Jacobi identity on $[\cdot, \cdot]_{\mathfrak{g}}$. For $g \neq 1$, (iii) can be rewritten as

$$
0=\sum_{\sigma \in \mathrm{Alt}_{3}} a_{g}\left(v_{\sigma(2)}, v_{\sigma(3)}\right)\left({ }^{g} v_{\sigma(1)}-v_{\sigma(1)}\right),
$$

which is the second part of condition 2 in the statement of the proposition. Finally, Theorem 3.1(iv) reduces to condition 3. In particular, when $\mathscr{H}$ satisfies the PBW condition, we may view the vector space $V$ as a Lie algebra $\mathfrak{g}$ under a Lie bracket $[\cdot, \cdot]_{\mathfrak{g}} \cdot$

In the nonmodular setting, we may use previous analysis of Drinfeld Hecke algebras (i.e., graded Hecke algebras) to interpret the conditions in the proposition in some detail. Conditions on the functions $a_{g}$ result from a comparison of condition 2 in the proposition and the invariance condition of Theorem 3.1(i) with [Ram and Shepler 2003, Lemma 1.5, equations (1.6) and (1.7), and Theorem 1.9]: If char( $k$ ) does not divide $|G|$, then the Jacobi condition 2 in Proposition 4.1 is equivalent to the condition that for each $g \neq 1$, either $a_{g} \equiv 0$ or $\operatorname{Ker} a_{g}=V^{g}$ with $\operatorname{codim}\left(V^{g}\right)=2$.

We further interpret the restrictive condition 3 in Proposition 4.1: Fix $g$ in $G$ with $\operatorname{codim} V^{g}=2$. Choose vectors $v_{1}, v_{2}$ spanning $\left(V^{g}\right)^{\perp}$ and $v_{3}, \ldots, v_{n}$ spanning $V^{g}$. This condition then tells us that after expanding with respect to the basis $v_{1}, \ldots, v_{n}$, the coefficient of $v_{2}$ in $\left[v_{2}, v_{i}\right]_{\mathfrak{g}}$ is equal to the coefficient of $v_{1}$ in $\left[v_{i}, v_{1}\right]_{\mathfrak{g}}$ for all $i \geq 3$. 
Remark 4.2. We view Lie orbifold algebras as generalizations both of symplectic reflection algebras and of universal enveloping algebras with group actions. Indeed, when $\mathscr{H}$ is a Lie orbifold algebra, we can replace each function $a_{g}$ with the zero function and recover the skew group algebra $U \# G$ for $G$ acting as automorphisms on $\mathcal{U}$, the universal enveloping algebra of a finite dimensional Lie algebra. Alternatively, we can replace the linear parameter by zero, i.e., replace $[\cdot, \cdot]_{\mathfrak{g}}$ by the zero bracket, and recover a Drinfeld Hecke algebra (a symplectic reflection algebra in the special case that $G$ acts symplectically). Thus, Lie orbifold algebras also include Drinfeld Hecke algebras (and Lusztig's graded affine Hecke algebra, in particular) as special cases. We illustrate by giving details for the example mentioned in the introduction.

Example 4.3. Let $\mathfrak{g}=\mathfrak{s l}_{2}$ over $\mathbb{C}$ with basis $e, f, h$ and Lie bracket defined by

$$
[e, f]=h, \quad[h, e]=2 e, \quad[h, f]=-2 f .
$$

Let $G$ be a cyclic group of order 2 generated by $g$ acting on $\mathfrak{s l}_{2}$ by

$$
{ }^{g} e=f, \quad{ }^{g} f=e, \quad{ }^{g} h=-h .
$$

The bracket is $G$-invariant under this action. Let $a_{g}$ be the skew-symmetric form on $V=\mathfrak{s l}_{2}$ defined by

$$
a_{g}(e, h)=1, \quad a_{g}(h, f)=1, \quad a_{g}(f, e)=0 .
$$

This function is $G$-invariant, and $\operatorname{Ker} a_{g}=V^{g}$ is the linear span of $e+f$, which has codimension 2 in $V$. Furthermore, $a_{g}$ is compatible with the Lie bracket, that is, condition 3 of Proposition 4.1 holds. (It suffices to check this condition for $v_{1}=e$, $v_{2}=f, v_{3}=h$ :

$$
\left.a_{g}(h,[e, f])+a_{g}(e,[f, h])+a_{g}(f,[h, e])=0 .\right)
$$

Set $a_{1}$ equal to the zero function. Then $T(V) \# G$ modulo the ideal generated by

$$
e h-h e+2 e-g, h f-f h+2 f-g, \quad e f-f e-h
$$

is a Lie orbifold algebra.

In fact, Theorem 3.1 shows there are only two parameters' worth of Lie orbifold algebras capturing this action of $G$ on $\mathfrak{s l}_{2}$ : Every such Lie orbifold algebra has the form

$$
T(V) \# G /\left(e h-h e+2 e-t_{2} g+t_{1}, h f-f h+2 f-t_{2} g+t_{1}, e f-f e-h\right)
$$

for some scalars $t_{1}, t_{2}$ in $\mathbb{C}$. (Note that $t_{1}=t_{2}=0$ defines the universal enveloping algebra extended by $G$.) 


\section{Koszul resolution}

Hochschild cohomology catalogues and illuminates deformations of an algebra. Indeed, every deformation of a $k$-algebra $R$ corresponds to an element in degree 2 Hochschild cohomology, $\mathrm{HH}^{2}(R)$. Isomorphic deformations define cohomologous cocycles. We isolated in [Shepler and Witherspoon 2008] the cocycles that define Drinfeld Hecke algebras (i.e., graded Hecke algebras): Drinfeld Hecke algebras are precisely those deformations of $S \# G$ whose corresponding Hochschild 2-cocycles are "constant". In the next section, we explain this statement, and we more generally express conditions for a quotient of $T(V) \# G$ to define a Drinfeld orbifold algebra in terms of the Hochschild cohomology of $S \# G$ and its graded Lie structure. Here we establish preliminaries and notation. From now on, we assume that the characteristic of $k$ does not divide the order of $G$ and that $k$ contains the eigenvalues of the actions of elements of $G$ on $V$. (For example, take $k$ algebraically closed of characteristic coprime to $|G|$.)

Recall that we denote the image of $v$ in $V$ under the action of any $g$ in $G$ by ${ }^{g} v$. Write $V^{*}$ for the contragredient (or dual) representation. Given any basis $v_{1}, \ldots, v_{n}$ of $V$, let $v_{1}^{*}, \ldots, v_{n}^{*}$ denote the dual basis of $V^{*}$. Given any set $A$ carrying an action of $G$, we write $A^{G}$ for the subset of elements invariant under the action. Again, we write $V^{g}$ for the $g$-invariant subspace of $V$. Since $G$ is finite, we may assume $G$ acts by isometries on $V$ (i.e., $G$ preserves a Hermitian form on $V$ ).

The Hochschild cohomology $\mathrm{HH}^{\bullet}(S \# G)$ is the space $\operatorname{Ext}_{(S \# G)^{e}}(S \# G, S \# G)$, where $(S \# G)^{e}=(S \# G) \otimes(S \# G)^{\text {op }}$ acts on $S \# G$ by multiplication, one tensor factor acting on the left and the other tensor factor acting on the right. We also examine the Hochschild cohomology $\mathrm{HH}^{\bullet}(S, S \# G):=\operatorname{Ext}_{S^{e}}(S, S \# G)$ where $S^{e}=S \otimes S^{\text {op }}$ and, more generally, $\mathrm{HH}^{\bullet}(S, M):=\operatorname{Ext}_{S^{e}}(S, M)$ for any $S^{e}$-module $M$.

Let $\mathscr{C}$ be a set of representatives of the conjugacy classes of $G$. For any $g$ in $G$, let $Z(g)$ be the centralizer of $g$. Since we have assumed that the characteristic of $k$ does not divide the order of $G$, there is a $G$-action giving the first of the following isomorphisms of graded vector spaces (see, for example, Ştefan [1995, Corollary 3.4]):

$$
\begin{aligned}
\mathrm{HH}^{\bullet}(S \# G) & \cong \mathrm{HH}^{\bullet}(S, S \# G)^{G} \\
& \cong\left(\bigoplus_{g \in G} \mathrm{HH}^{\bullet}(S, S g)\right)^{G} \cong \bigoplus_{g \in \mathscr{C}} \mathrm{HH}^{\bullet}(S, S g)^{Z(g)} .
\end{aligned}
$$

The first line is in fact a graded algebra isomorphism; it follows from applying a spectral sequence. The second isomorphism results from decomposing the bimodule $S \# G$ into the direct sum of components $S g$. The action of $G$ permutes these components via the conjugation action of $G$ on itself, and thus the third 
isomorphism is a canonical projection onto a set of representative summands. Each space $\mathrm{HH}^{\bullet}(S, S g)=\operatorname{Ext}_{S^{e}}(S, S g)$ may be determined explicitly using the Koszul resolution of $S$ (a free resolution of $S$ as an $S^{e}$-module) that we recall next.

The Koszul resolution $K_{\bullet}(S)$ is defined by $K_{0}(S)=S^{e}$ and

$$
K_{p}(S)=S^{e} \otimes \bigwedge^{p}(V)
$$

for $p \geq 1$, with differentials

$$
\begin{aligned}
d_{p}\left(1 \otimes 1 \otimes v_{j_{1}}\right. & \left.\wedge \cdots \wedge v_{j_{p}}\right) \\
& =\sum_{i=1}^{p}(-1)^{i+1}\left(v_{j_{i}} \otimes 1-1 \otimes v_{j_{i}}\right) \otimes\left(v_{j_{1}} \wedge \cdots \wedge \hat{v}_{j_{i}} \wedge \cdots \wedge v_{j_{p}}\right)
\end{aligned}
$$

for all $v_{j_{1}}, \ldots, v_{j_{p}} \in V$ (e.g., see [Weibel 1994, §4.5]). We apply $\operatorname{Hom}_{S^{e}}(-, S g$ ) to each term of the Koszul resolution and then make the identifications

$$
\operatorname{Hom}_{S^{e}}\left(S^{e} \otimes \bigwedge^{p}(V), S g\right) \cong \operatorname{Hom}_{k}\left(\bigwedge^{p} V, S g\right) \cong S g \otimes \bigwedge^{p} V^{*}
$$

for each $g$ in $G$. Thus we write the set of cochains arising from the Koszul resolution (from which the cohomology classes emerge) as vector forms on $V$ tagged by group elements: Let

$$
C^{\bullet}=\bigoplus_{g \in G} C_{g}^{\bullet}, \quad \text { where } \quad C_{g}^{p}:=S g \otimes \wedge^{p} V^{*} \quad \text { for each } g \in G .
$$

We call $C_{g}^{\bullet}$ the space of cochains supported on $g$. Similarly, for any subset $X$ of $G$, we define $C_{X}^{\bullet}:=\oplus_{g \in X} C_{g}^{\bullet}$, the set of cochains supported on $X$. We say a cochain in $C^{\bullet}$ is supported off a subset $X$ of $G$ if it lies in $\oplus_{g \notin X} C_{g}^{\bullet}$. Note that each element of $G$ permutes the summands of $C^{\bullet}$ via the conjugation action of $G$ on itself.

From the space $C^{\bullet}$ of cochains, we define a space of representatives of cohomology classes: Let

$$
H^{\bullet}:=\bigoplus_{g \in G} S\left(V^{g}\right) g \otimes \bigwedge^{\bullet-\operatorname{codim} V^{g}}\left(V^{g}\right)^{*} \otimes \bigwedge^{\operatorname{codim} V^{g}}\left(\left(V^{g}\right)^{\perp}\right)^{*} .
$$

Then $H^{\bullet} \subset C^{\bullet}$ with

$$
H^{\bullet} \cong \mathrm{HH}^{\bullet}(S, S \# G) \text { and }\left(H^{\bullet}\right)^{G} \cong \mathrm{HH}^{\bullet}(S, S \# G)^{G} \cong \mathrm{HH}^{\bullet}(S \# G) .
$$

(See [Shepler and Witherspoon 2012, Proposition 5.11 and (6.1)] for this formulation of the Hochschild cohomology. It was first computed independently in [Farinati 2005] and [Ginzburg and Kaledin 2004].) In particular it follows that $\left(H^{2}\right)^{G}$ is supported on elements $g$ for which codim $V^{g} \in\{0,2\}$, since an element of $\left(H^{2}\right)^{G}$ is invariant under the action of each group element $g$. See [Shepler and Witherspoon 2008, Lemma 3.6] for details. 
The grading on the polynomial ring $S=S(V)$ induces a grading on the set of cochains by polynomial degree: We say a cochain in $C^{\bullet}$ has polynomial degree $i$ if the factors in $S$ in the expression (5.4) are all polynomials of degree $i$. We say a cochain is homogeneous when its polynomial factors in $S$ are homogeneous. A constant cochain is then one of polynomial degree 0 and a linear cochain is one of homogeneous polynomial degree 1 . The cochains $C^{\bullet}$ are filtered by polynomial degree: $C_{0}^{\bullet} \subset C_{1}^{\bullet} \subset C_{2}^{\bullet} \subset \cdots$, where $C_{i}^{\bullet}$ is the subspace of $C^{\bullet}$ consisting of cochains of polynomial degree at most $i$.

Definition 5.6. We define a cochain bracket map on the subspace generated by linear and constant 2-cochains: Let $[\cdot, \cdot]: C_{1}^{2} \times C_{1}^{2} \rightarrow C_{1}^{3}$ be the symmetric map defined by setting $[\alpha, \beta]\left(v_{1}, v_{2}, v_{3}\right)$ (for any $v_{1}, v_{2}, v_{3}$ in $V$ ) to

$$
\sum_{\substack{g, h \in G \\ \sigma \in \mathrm{Alt}_{3}}}\left[\alpha_{g h^{-1}}\left(\beta_{h}\left(v_{\sigma(1)} \wedge v_{\sigma(2)}\right) \wedge v_{\sigma(3)}\right)+\beta_{g h^{-1}}\left(\alpha_{h}\left(v_{\sigma(1)} \wedge v_{\sigma(2)}\right) \wedge v_{\sigma(3)}\right)\right] g
$$

if $\alpha$ and $\beta$ are linear, to

$$
\sum_{\substack{g, h \in G \\ \sigma \in \mathrm{Alt}_{3}}} \alpha_{g h^{-1}}\left(\beta_{h}\left(v_{\sigma(1)} \wedge v_{\sigma(2)}\right) \wedge v_{\sigma(3)}\right) g
$$

if $\alpha$ is constant and $\beta$ is linear, and to 0 for constant $\alpha$ and $\beta$.

We will see in the next section that this definition gives a representative cochain for a class in cohomology $\mathrm{HH}^{\bullet}(S \# G)$ of the Gerstenhaber bracket of $\alpha$ and $\beta$ when they are cocycles.

\section{Gerstenhaber bracket}

In this section we recall the definition of the Gerstenhaber bracket on Hochschild cohomology, defined on the bar resolution, and show how it is related to the cochain bracket map of Definition 5.6. Recall the definition of the bar resolution of a $k$-algebra $R$ : It has $p$ th term $R^{\otimes(p+2)}$ and differentials

$$
\delta_{p}\left(r_{0} \otimes \cdots \otimes r_{p+1}\right)=\sum_{i=0}^{p}(-1)^{i} r_{0} \otimes \cdots \otimes r_{i} r_{i+1} \otimes \cdots \otimes r_{p+1}
$$

for all $r_{0}, \ldots, r_{p+1} \in R$. From this one may derive the standard definition of a Hochschild 2-cocycle: It is an element $\mu$ of $\operatorname{Hom}_{k}(R \otimes R, R) \cong \operatorname{Hom}_{R^{e}}\left(R^{\otimes 4}, R\right)$ for which

$$
\mu(r s, u)+\mu(r, s) u=\mu(r, s u)+r \mu(s, u)
$$

for all $r, s, u \in R$. (Here we have further identified the linear map $\mu$ on $R \otimes R$ with a bilinear map on $R \times R$.) 
Lemma 6.2. Let $\mu$ be a Hochschild 2-cocycle on $S \# G$ whose kernel contains $k G$. Then

$$
\mu(r g, s)=\mu(r, g s)=\mu\left(r,{ }^{g} s\right) g
$$

for all $r, s$ in $S$ and $g$ in $G$.

Proof. Apply (6.1) to $r, g, s$ to obtain $\mu(r g, s)+\mu(r, g) s=\mu(r, g s)+r \mu(g, s)$. By hypothesis, $\mu(r, g)=0=\mu(g, s)$, so $\mu(r g, s)=\mu(r, g s)$. Now apply (6.1) to $r$, ${ }^{g} s, g$ to obtain $\mu\left(r\left({ }^{g} s\right), g\right)+\mu\left(r,{ }^{g} s\right) g=\mu\left(r,\left({ }^{g} s\right) g\right)+r \mu\left({ }^{g} s, g\right)$. By hypothesis, $\mu\left(r\left({ }^{g} s\right), g\right)=0=\mu\left({ }^{g} s, g\right)$, so $\mu\left(r,{ }^{g} s\right) g=\mu\left(r,\left({ }^{g} s\right) g\right)$. Since $g s=\left({ }^{g} s\right) g$, the lemma follows.

We will also need the definition of the circle operation on Hochschild cohomology in degree 2: If $R$ is a $k$-algebra and $\alpha$ and $\beta$ are elements of $\operatorname{Hom}_{R^{e}}\left(R^{\otimes 4}, R\right) \cong$ $\operatorname{Hom}_{k}\left(R^{\otimes 2}, R\right)$, then $\alpha \circ \beta \in \operatorname{Hom}_{k}\left(R^{\otimes 3}, R\right)$ is defined by

$$
\alpha \circ \beta\left(r_{1} \otimes r_{2} \otimes r_{3}\right):=\alpha\left(\beta\left(r_{1} \otimes r_{2}\right) \otimes r_{3}\right)-\alpha\left(r_{1} \otimes \beta\left(r_{2} \otimes r_{3}\right)\right)
$$

for all $r_{1}, r_{2}, r_{3} \in R$. The Gerstenhaber bracket is then

$$
[\alpha, \beta]:=\alpha \circ \beta+\beta \circ \alpha .
$$

This bracket is well-defined on cohomology classes, but the circle operation is not. (See [Gerstenhaber 1963, §7] for the circle operation and brackets in other degrees.) In our setting, $R=S \# G$, and we now express the Gerstenhaber bracket on input from the Koszul resolution using the cochain bracket of Definition 5.6. In the theorem below, we fix a choice of isomorphism $\mathrm{HH}^{\bullet}(S \# G) \cong\left(H^{\bullet}\right)^{G}$ where $H^{\bullet}$ is given by (5.5). (See [Shepler and Witherspoon 2012, Proposition 5.11 and (6.1)].)

Theorem 6.3. Consider two cohomology classes $\alpha^{\prime}, \beta^{\prime}$ in $\mathrm{HH}^{2}(S \# G)$ represented by cochains $\alpha, \beta$ in $\left(H^{2}\right)^{G}$ of polynomial degree at most 1 . Then the Gerstenhaber bracket in $\mathrm{HH}^{3}(S \# G)$ of $\alpha^{\prime}$ and $\beta^{\prime}$ is represented by the cochain bracket $[\alpha, \beta]$ of Definition 5.6.

Proof. We use the chain map $\phi$. from the Koszul resolution $K .(S)$ to the bar resolution for $S=S(V)$ given in each degree by

$$
\phi_{p}\left(1 \otimes 1 \otimes v_{j_{1}} \wedge \cdots \wedge v_{j_{p}}\right)=\sum_{\sigma \in \operatorname{Sym}_{p}} \operatorname{sgn}(\sigma) \otimes v_{j_{\sigma(1)}} \otimes \cdots \otimes v_{j_{\sigma(p)}} \otimes 1
$$

for all $v_{j_{1}}, \ldots, v_{j_{p}} \in V$, where $\operatorname{Sym}_{p}$ denotes the symmetric group on $p$ symbols. We may view functions on the bar resolution in cohomological degree 2 as functions on $K_{2}(S)=S^{e} \otimes \bigwedge^{2}(V)$ simply by composing with $\phi_{2}$.

We will also need a choice $\psi$. of chain map from the bar to the Koszul resolution. The particular choice of $\psi$. does not matter here, but we will assume that $\psi \phi$ is the identity map and that $\psi_{2}(1 \otimes a \otimes b \otimes 1)=0$ if either $a$ or $b$ is in the field $k$. 
(For example, one could take $\psi_{\bullet}$ so that $\psi_{2}\left(1 \otimes v_{i} \otimes v_{j} \otimes 1\right)=1 \otimes 1 \otimes v_{i} \wedge v_{j}$ for $i<j$ and 0 otherwise, for some fixed basis $v_{1}, \ldots, v_{n}$ of $V$. See [Shepler and Witherspoon 2011] for explicit constructions of such maps $\psi_{\bullet}$; we will not need them here.) Note that although $\psi_{2}$ may not be a $k G$-homomorphism, the map $\psi_{2}$ preserves the action of $G$ on the image of $\phi_{2}$. For our purposes here, this implies that we do not need to average over $G$ when computing brackets, as is done, for example, in [Shepler and Witherspoon 2012]. We also note that every chain map $\psi$ for which $\psi \phi$ is the identity map has the property that

$$
\psi_{2}(1 \otimes v \otimes w \otimes 1-1 \otimes w \otimes v \otimes 1)=1 \otimes 1 \otimes v \wedge w
$$

for all $v, w$ in $V$. Thus on elements of this form, $\psi_{2}$ is independent of choice of basis of $V$.

We extend each function $\gamma$ on the bar complex for $S$ to a function on the bar complex for $S \# G$ in a standard way: In degree 2 , we require $k G$ to be in the kernel of $\gamma$ and set

$$
\gamma\left(1 \otimes s_{1} g_{1} \otimes s_{2} g_{2} \otimes 1\right):=\gamma\left(1 \otimes s_{1} \otimes{ }^{g_{1}} s_{2} \otimes 1\right) g_{1} g_{2}
$$

for $\gamma \in \operatorname{Hom}_{S^{e}}\left(S^{\otimes 4}, S \# G\right), s_{1}, s_{2} \in S, g_{1}, g_{2} \in G$. Compare with Lemma 6.2. (See [Căldăraru et al. 2004, Theorem 5.4] for a more general statement.)

We apply the chain map $\psi$ to convert $\alpha$ and $\beta$ to functions on the bar complex, execute the Gerstenhaber bracket, and apply $\phi$ to convert back to a function on the Koszul complex. The induced operation on cochains arising from the Koszul complex is thus

$$
[\alpha, \beta]:=\phi^{*}\left(\psi^{*}(\alpha) \circ \psi^{*}(\beta)\right)+\phi^{*}\left(\psi^{*}(\beta) \circ \psi^{*}(\alpha)\right) .
$$

Note there is no guarantee that $[\alpha, \beta]$ is in the chosen space $H^{3}$ of representatives of cohomology classes, however there is a unique element of $H^{3}$ to which it is cohomologous.

We compute separately the two corresponding circle operations, keeping in mind that they are not well-defined on cohomology, and so must be combined. (Again, we identify $\operatorname{Hom}_{S^{e}}\left(S^{\otimes(p+2)}, *\right)$ with $\operatorname{Hom}_{k}\left(S^{\otimes p}, *\right)$ and $\operatorname{Hom}_{S^{e}}\left(S^{e} \otimes \bigwedge^{p} V, *\right)$ with $\operatorname{Hom}_{k}\left(\bigwedge^{p} V, *\right)$, dropping extra tensor factors of 1.) Then

$$
\begin{aligned}
& (\alpha \circ \beta)\left(v_{1} \wedge v_{2} \wedge v_{3}\right) \\
& =\left(\psi^{*}(\alpha) \circ \psi^{*}(\beta)\right) \phi\left(v_{1} \wedge v_{2} \wedge v_{3}\right) \\
& =\left(\psi^{*}(\alpha) \circ \psi^{*}(\beta)\right) \sum_{\sigma \in S_{3}} \operatorname{sgn} \sigma v_{\sigma(1)} \otimes v_{\sigma(2)} \otimes v_{\sigma(3)} \\
& =\sum_{\sigma \in S_{3}} \operatorname{sgn} \sigma \psi^{*}(\alpha)\left(\psi^{*}(\beta)\left(v_{\sigma(1)} \otimes v_{\sigma(2)}\right) \otimes v_{\sigma(3)}-v_{\sigma(1)} \otimes \psi^{*}(\beta)\left(v_{\sigma(2)} \otimes v_{\sigma(3)}\right)\right) .
\end{aligned}
$$


We may rewrite the sum over the alternating group instead to obtain

$$
\begin{aligned}
& (\alpha \circ \beta)\left(v_{1} \wedge v_{2} \wedge v_{3}\right) \\
& =\sum_{\sigma \in \mathrm{Alt}_{3}} \psi^{*}(\alpha)\left(\psi^{*}(\beta)\left(v_{\sigma(1)} \otimes v_{\sigma(2)}\right) \otimes v_{\sigma(3)}-\psi^{*}(\beta)\left(v_{\sigma(2)} \otimes v_{\sigma(1)}\right) \otimes v_{\sigma(3)}\right) \\
& \quad-\psi^{*}(\alpha)\left(v_{\sigma(1)} \otimes \psi^{*}(\beta)\left(v_{\sigma(2)} \otimes v_{\sigma(3)}\right)-v_{\sigma(1)} \otimes \psi^{*}(\beta)\left(v_{\sigma(3)} \otimes v_{\sigma(2)}\right)\right) .
\end{aligned}
$$

But $\psi^{*}(\beta)(v \otimes w-w \otimes v)=\beta(v \wedge w)$ for all vectors $v, w$ in $V$, and hence Lemma 6.2 implies that the sum above is just

$$
\begin{aligned}
& \sum_{\sigma \in \mathrm{Alt}_{3}} \psi^{*}(\alpha)\left(\beta\left(v_{\sigma(1)} \wedge v_{\sigma(2)}\right) \otimes v_{\sigma(3)}-v_{\sigma(1)} \otimes \beta\left(v_{\sigma(2)} \wedge v_{\sigma(3)}\right)\right) \\
= & \sum_{\substack{\sigma \in \mathrm{Alt}_{3} \\
h \in G}} \psi^{*}(\alpha)\left(\beta_{h}\left(v_{\sigma(1)} \wedge v_{\sigma(2)}\right) h \otimes v_{\sigma(3)}-v_{\sigma(1)} \otimes \beta_{h}\left(v_{\sigma(2)} \wedge v_{\sigma(3)}\right) h\right) \\
= & \sum_{\substack{\sigma \in \mathrm{Alt}_{3} \\
h \in G}} \psi^{*}(\alpha)\left(\beta_{h}\left(v_{\sigma(1)} \wedge v_{\sigma(2)}\right) \otimes^{h} v_{\sigma(3)}-v_{\sigma(1)} \otimes \beta_{h}\left(v_{\sigma(2)} \wedge v_{\sigma(3)}\right)\right) h .
\end{aligned}
$$

First assume the polynomial degree of $\beta$ is 0 . Then each $\beta_{h}\left(v_{i} \wedge v_{j}\right)$ is constant. But $\psi^{*}(\alpha)(a \otimes b)$ is zero for either $a$ or $b$ in $k$, and the last expression is thus zero. Hence, $\alpha \circ \beta\left(v_{1} \wedge v_{2} \wedge v_{3}\right)$ is zero for $\beta$ of polynomial degree 0 .

Now assume $\beta$ is homogeneous of polynomial degree 1. We claim that for any $h$ in $G$ and any $u_{1}, u_{2}, u_{3}$ in $V$,

$$
\sum_{\sigma \in \mathrm{Alt}_{3}} \beta_{h}\left(u_{\sigma(1)} \wedge u_{\sigma(2)}\right) \otimes{ }^{h} u_{\sigma(3)}=\sum_{\sigma \in \mathrm{Alt}_{3}} \beta_{h}\left(u_{\sigma(1)} \wedge u_{\sigma(2)}\right) \otimes u_{\sigma(3)} .
$$

The equation clearly holds for $h$ acting trivially on $V$. One may easily verify the equation for $h$ not in the kernel of the representation $G \rightarrow \mathrm{GL}(V)$ by fixing a basis of $V$ consisting of eigenvectors for $h$ and using the fact that any nonzero $\beta_{h}$ is supported on $\bigwedge^{2}\left(V^{h}\right)^{\perp}$ with codim $V^{h}=2$; see (5.5).

We use (6.6) and the fact that $\psi^{*}(\alpha)(v \otimes w-w \otimes v)=\alpha(v \wedge w)$ for all vectors $v, w$ in $V$ to simplify (6.5):

$$
\begin{aligned}
& (\alpha \circ \beta)\left(v_{1} \wedge v_{2} \wedge v_{3}\right) \\
& =\sum_{\substack{\sigma \in \mathrm{Alt}_{3} \\
h \in G}} \psi^{*}(\alpha)\left(\beta_{h}\left(v_{\sigma(1)} \wedge v_{\sigma(2)}\right) \otimes{ }^{h} v_{\sigma(3)}-v_{\sigma(1)} \otimes \beta_{h}\left(v_{\sigma(2)} \wedge v_{\sigma(3)}\right)\right) h \\
& =\sum_{\substack{\sigma \in \mathrm{Alt}_{3} \\
h \in G}} \psi^{*}(\alpha)\left(\beta_{h}\left(v_{\sigma(1)} \wedge v_{\sigma(2)}\right) \otimes v_{\sigma(3)}-v_{\sigma(1)} \otimes \beta_{h}\left(v_{\sigma(2)} \wedge v_{\sigma(3)}\right)\right) h \\
& =\sum_{\substack{\sigma \in \mathrm{Alt}_{3} \\
h \in G}} \psi^{*}(\alpha)\left(\beta_{h}\left(v_{\sigma(1)} \wedge v_{\sigma(2)}\right) \otimes v_{\sigma(3)}-v_{\sigma(3)} \otimes \beta_{h}\left(v_{\sigma(1)} \wedge v_{\sigma(2)}\right)\right) h
\end{aligned}
$$




$$
\begin{aligned}
& =\sum_{\substack{\sigma \in \mathrm{Alt}_{3} \\
h \in G}} \alpha\left(\beta_{h}\left(v_{\sigma(1)} \wedge v_{\sigma(2)}\right) \wedge v_{\sigma(3)}\right) h \\
& =\sum_{\substack{\sigma \in \mathrm{Alt}_{3} \\
g, h \in G}} \alpha_{g}\left(\beta_{h}\left(v_{\sigma(1)} \wedge v_{\sigma(2)}\right) \wedge v_{\sigma(3)}\right) g h .
\end{aligned}
$$

A similar computation for $\beta \circ \alpha$ together with reindexing over the group yields the result.

The Gerstenhaber bracket takes a particularly nice form when we consider square brackets of linear cocycles and brackets of linear with constant cocycles:

Corollary 6.8. Consider cohomology classes $\alpha^{\prime}, \beta^{\prime}$ in $\mathrm{HH}^{2}(S \# G)$ represented by a constant cocycle $\alpha$ and a linear cocycle $\beta$ in $\left(H^{2}\right)^{G}$, respectively. The Gerstenhaber bracket in $\mathrm{HH}^{3}(S \# G)$ of $\beta^{\prime}$ with itself is represented by the cocycle

$$
[\beta, \beta]\left(v_{1}, v_{2}, v_{3}\right)=2 \sum_{\substack{g, h \in G \\ \sigma \in \mathrm{Alt}_{3}}} \beta_{g h^{-1}}\left(\beta_{h}\left(v_{\sigma(1)} \wedge v_{\sigma(2)}\right) \wedge v_{\sigma(3)}\right) g
$$

that of $\alpha^{\prime}$ with $\beta^{\prime}$ is represented by the cocycle

$$
[\alpha, \beta]\left(v_{1}, v_{2}, v_{3}\right)=\sum_{\substack{g, h \in G \\ \sigma \in \mathrm{Alt}_{3}}} \alpha_{g h^{-1}}\left(\beta_{h}\left(v_{\sigma(1)} \wedge v_{\sigma(2)}\right) \wedge v_{\sigma(3)}\right) g .
$$

\section{PBW condition and Gerstenhaber bracket}

In this section, we give necessary and sufficient conditions on a parameter to define a Drinfeld orbifold algebra in terms of Hochschild cohomology. We interpret Theorem 3.1 in terms of cocycles and the Gerstenhaber bracket in cohomology as realized on the set of cochains arising from the Koszul resolution. Our results should be compared with [Halbout et al. 2011, §2.2, (4), (5), (6)], where a factor of 2 is missing from the right side of (5). See also [Khare 2007, (1.9)] for a somewhat different setting.

We want to describe precisely which parameter maps $\kappa$ result in a quotient $\mathscr{H}_{\kappa}$ that satisfies the PBW condition, that is, defines a Drinfeld orbifold algebra. The algebras $\mathscr{H}_{\kappa}$ are naturally expressed and analyzed in terms of the Koszul resolution of $S$. Recall, $\kappa: \bigwedge^{2} V \rightarrow S \otimes \mathbb{C} G$ with $\kappa=\sum_{g \in G} \kappa_{g} g$. The parameter map $\kappa$ as well as its linear and constant parts, $\kappa^{L}$ and $\kappa^{C}$, thus define cochains on the Koszul resolution and we identify $\kappa, \kappa^{L}, \kappa^{C}$ with elements of $C^{\bullet}$. Indeed, for each $g \in G$, the functions $\kappa_{g} g, \kappa_{g}^{L} g$, and $\kappa_{g}^{C} g$ (from $\wedge^{2} V$ to $S g$ ) define elements of the cochain complex $C_{g}^{\bullet}$ of (5.4).

We now determine a complete set of necessary and sufficient conditions on these parameters regarded as cochains in Hochschild cohomology $\mathrm{HH}^{\bullet}(S \# G) \cong$ $\mathrm{HH}^{\bullet}(S, S \# G)^{G}$. (We use the chain maps converting between resolutions discussed 
in Section 6.) The significance of the following lemma and theorem thereafter lies in the expression of the PBW property in terms of the Gerstenhaber bracket in cohomology. We distinguish a cochain $[\alpha, \beta]$ on the Koszul resolution (5.2) from its cohomology class arising from the induced Gerstenhaber bracket by using the phrase "as a cochain" where appropriate. (Recall that $d$ is the differential on the Koszul resolution defined as in (5.3).)

Lemma 7.1. In Theorem 3.1,

- condition (ii) holds if and only if $\kappa^{L}$ is a cocycle, i.e., $d^{*} \kappa^{L}=0$;

- for $\kappa^{L}$ in $H^{\bullet}$, condition (iii) is equivalent to $\left[\kappa^{L}, \kappa^{L}\right]=2 d^{*} \kappa^{C}$ as cochains;

- for $\kappa^{L}$ in $H^{\bullet}$, condition (iv) is equivalent to $\left[\kappa^{C}, \kappa^{L}\right]=0$ as a cochain.

Proof. The cochain $d^{*} \kappa^{L}$ is zero exactly when $\kappa^{L}$ takes to 0 all input of the form

$$
\begin{aligned}
d_{3}\left(v_{1} \wedge v_{2} \wedge v_{3}\right)= & \left(v_{1} \otimes 1-1 \otimes v_{1}\right) \otimes v_{2} \wedge v_{3} \\
- & \left(v_{2} \otimes 1-1 \otimes v_{2}\right) \otimes v_{1} \wedge v_{3} \\
+ & \left(v_{3} \otimes 1-1 \otimes v_{3}\right) \otimes v_{1} \wedge v_{2},
\end{aligned}
$$

in other words, when

$$
\begin{aligned}
0= & v_{1} \kappa^{L}\left(v_{2}, v_{3}\right)-\kappa^{L}\left(v_{2}, v_{3}\right) v_{1} \\
+ & v_{2} \kappa^{L}\left(v_{3}, v_{1}\right)-\kappa^{L}\left(v_{3}, v_{1}\right) v_{2} \\
+ & v_{3} \kappa^{L}\left(v_{1}, v_{2}\right)-\kappa^{L}\left(v_{1}, v_{2}\right) v_{3}
\end{aligned}
$$

in $S \# G$. This is equivalent to

$$
\begin{aligned}
0= & v_{1} \kappa_{g}^{L}\left(v_{2}, v_{3}\right) g-\kappa_{g}^{L}\left(v_{2}, v_{3}\right) g v_{1} \\
& +v_{2} \kappa_{g}^{L}\left(v_{3}, v_{1}\right) g-\kappa_{g}^{L}\left(v_{3}, v_{1}\right) g v_{2} \\
& +v_{3} \kappa_{g}^{L}\left(v_{1}, v_{2}\right) g-\kappa_{g}^{L}\left(v_{1}, v_{2}\right) g v_{3}
\end{aligned}
$$

for each $g$ in $G$. We rewrite this expression using the commutativity of $S$ and moving all factors of $g$ to the right:

$$
0=\kappa_{g}^{L}\left(v_{2}, v_{3}\right)\left(v_{1}-{ }^{g} v_{1}\right)+\kappa_{g}^{L}\left(v_{3}, v_{1}\right)\left(v_{2}-{ }^{g} v_{2}\right)+\kappa_{g}^{L}\left(v_{1}, v_{2}\right)\left(v_{3}-{ }^{g} v_{3}\right),
$$

which is precisely Theorem 3.1(ii).

Next, notice that we may apply Equation (6.6) (in the proof of Theorem 6.3) to $\beta=\kappa^{L}$, under the assumption that $\kappa^{L}$ lies in $H^{2}$. Then for each $g$ in $G$, the left side of Theorem 3.1(iii) is the opposite of the coefficient of $g$ in $\left[\kappa^{L}, \kappa^{L}\right]$ by Definition 5.6 (see Corollary 6.8) and the skew-symmetry of $\kappa^{L}$. By a similar calculation to that for $\kappa^{L}$, the right side of (iii) is the coefficient of $g$ in

$$
-2 d_{3}^{*} \kappa^{C}\left(v_{1} \wedge v_{2} \wedge v_{3}\right)=2 \sum_{g \in G} \sum_{\sigma \in \mathrm{Alt}_{3}} \kappa_{g}^{C}\left(v_{\sigma(2)}, v_{\sigma(3)}\right)\left({ }^{g} v_{\sigma(1)}-v_{\sigma(1)}\right) g .
$$


Hence, Theorem 3.1(iii) is equivalent to $\left[\kappa^{L}, \kappa^{L}\right]=2 d_{3}^{*} \kappa^{C}$. This condition differs from [Halbout et al. 2011, (5)] where the factor of 2 is missing.

We again compare coefficients of fixed $g$ in $G$ and apply Equation (6.6) to see that Theorem 3.1(iv) is equivalent to $\left[\kappa^{C}, \kappa^{L}\right]=0$ by Definition 5.6. Note that this is equivalent to [Halbout et al. 2011, (6)] when $k=\mathbb{R}$.

We are now ready to express the PBW property purely in cohomological terms. Recall that $H^{\bullet}$ is the fixed space of representatives of elements in $\mathrm{HH}^{\bullet}(S, S \# G)$ defined in (5.5).

Theorem 7.2. A quotient algebra $\mathscr{H}_{\tilde{\kappa}}$ is a Drinfeld orbifold algebra if and only if $\mathscr{H}_{\tilde{\kappa}}$ is isomorphic to $\mathscr{H}_{\kappa}$ as a filtered algebra for some parameter $\kappa$ satisfying these conditions:

1. $\kappa$ is $G$-invariant.

2. The linear part of $\kappa$ is a cocycle in $H^{\bullet}$.

3. The Gerstenhaber square bracket of the linear part of $\kappa$ satisfies $\left[\kappa^{L}, \kappa^{L}\right]=$ $2 d^{*}\left(\kappa^{C}\right)$ as cochains.

4. The bracket of the linear with the constant part of $\kappa$ is zero: $\left[\kappa^{C}, \kappa^{L}\right]=0$ as a cochain.

Proof. Write $\tilde{\kappa}=\tilde{\kappa}^{L}+\tilde{\kappa}^{C}$. If $\mathscr{H}_{\tilde{\kappa}}$ is a Drinfeld orbifold algebra, then it satisfies condition (ii) of Theorem 3.1. Lemma 7.1 then implies that $\tilde{\kappa}^{L}$ is a cocycle in $\mathrm{HH}^{\bullet}(S, S \# G)$ expressed with respect to the Koszul resolution, and it thus lies in the set of cohomology representatives $\left(H^{\bullet}\right)^{G}$ up to a coboundary:

$$
\tilde{\kappa}^{L}=\kappa^{L}+d^{*} \rho
$$

for some 2-cocycle $\kappa^{L}$ in $\left(H^{2}\right)^{G}$ and some 1-cochain $\rho$. Set

$$
\kappa^{C}=\tilde{\kappa}^{C}+\rho \circ \tilde{\kappa}^{L}-\rho \otimes \rho
$$

where $(\rho \otimes \rho)(v \wedge w):=\rho(v) \rho(w)-\rho(w) \rho(v)$ for all $v, w \in V$. Let $\kappa=\kappa^{C}+\kappa^{L}$.

We may assume without loss of generality that $\rho$ is $G$-invariant. (Note that $d^{*} \rho=\tilde{\kappa}^{L}-\kappa^{L}$ is $G$-invariant. Since $d^{*}$ commutes with the group action and the order of $G$ is invertible in $k$, we may replace $\rho$ by $\frac{1}{|G|} \sum_{g \in G}{ }^{g} \rho$ to obtain a cochain having the same image under $d^{*}$.) Also note that without loss of generality $\rho$ takes values in $k G$ since $d^{*} \rho$ has polynomial degree 1 .

Define a map $f: T(V) \# G \rightarrow \mathscr{H}_{\kappa}$ by

$$
f(v)=v+\rho(v), \quad f(g)=g
$$

for all $v \in V, g \in G$; since $\rho$ is $G$-invariant, these values extend uniquely to give an algebra homomorphism. Note that $f$ is surjective by an inductive argument on the degrees of elements. 
We show first that the kernel of $f$ contains the ideal

$$
\left(v w-w v-\tilde{\kappa}^{L}(v, w)-\tilde{\kappa}^{C}(v, w) \mid v, w \in V\right),
$$

which implies that $f$ induces an algebra homomorphism from $\mathscr{H}_{\tilde{\kappa}}$ onto $\mathscr{H}_{\kappa}$. By the definition of $f$,

$$
\begin{aligned}
& f\left(v w-w v-\tilde{\kappa}^{L}(v, w)-\tilde{\kappa}^{C}(v, w)\right) \\
& =(v+\rho(v))(w+\rho(w))-(w+\rho(w))(v+\rho(v)) \\
& \quad-\tilde{\kappa}^{L}(v, w)-\rho\left(\tilde{\kappa}^{L}(v, w)\right)-\tilde{\kappa}^{C}(v, w) \\
& =v w-w v+v \rho(w)+\rho(v) w-w \rho(v)-\rho(w) v+\rho(v) \rho(w)-\rho(w) \rho(v) \\
& \quad-\tilde{\kappa}^{L}(v, w)-\rho \circ \tilde{\kappa}^{L}(v, w)-\tilde{\kappa}^{C}(v, w) \\
& =v w-w v+d^{*} \rho(v \wedge w)+(\rho \otimes \rho)(v \wedge w)-\tilde{\kappa}^{L}(v, w)-\rho \circ \tilde{\kappa}^{L}(v, w)-\tilde{\kappa}^{C}(v, w) \\
& =v w-w v-\kappa^{L}(v, w)-\kappa^{C}(v, w)=0
\end{aligned}
$$

in $\mathscr{H}_{\kappa}$. Thus the ideal generated by all $v w-w v-\tilde{\kappa}^{L}(v, w)-\tilde{\kappa}^{C}(v, w)$ is in the kernel of $f$.

Next we define an inverse to $f$ by replacing $\rho$ with $-\rho$ : Define an algebra homomorphism $f^{\prime}: T(V) \# G \rightarrow \mathscr{H}_{\tilde{\kappa}}$ by $f^{\prime}(v)=v-\rho(v), f(g)=g$ for all $v \in V$, $g \in G$. We have $\kappa^{L}=\tilde{\kappa}^{L}-d^{*} \rho$ and

$$
\begin{aligned}
\tilde{\kappa}^{C} & =\kappa^{C}-\rho \circ \tilde{\kappa}^{L}+\rho \otimes \rho \\
& =\kappa^{C}-\rho \circ \kappa^{L}-\rho \circ\left(d^{*} \rho\right)+\rho \otimes \rho .
\end{aligned}
$$

Extending $\rho$ in the usual way from a function on $V$ to a function on $V \otimes k G$ by setting $\rho(v g):=\rho(v) g$ for all $v \in V, g \in G$, we calculate

$$
\begin{aligned}
\rho \circ\left(d^{*} \rho\right)(v \wedge w) & =\rho(v \rho(w)+\rho(v) w-w \rho(v)-\rho(w) v) \\
& =\rho(v) \rho(w)+\rho(v) \rho(w)-\rho(w) \rho(v)-\rho(w) \rho(v) \\
& =2(\rho \otimes \rho)(v \wedge w),
\end{aligned}
$$

since $\rho$ has image in $k G$. Thus we may rewrite

$$
\begin{aligned}
\tilde{\kappa}^{C} & =\kappa^{C}-\rho \circ \kappa^{L}-2 \rho \otimes \rho+\rho \otimes \rho \\
& =\kappa^{C}-\rho \circ \kappa^{L}-\rho \otimes \rho \\
& =\kappa^{C}+(-\rho) \circ \kappa^{L}-(-\rho) \otimes(-\rho) .
\end{aligned}
$$

An argument similar to that above for $f$ (replacing $\rho$ by $-\rho$ ) shows that the function $f^{\prime}$ induces an algebra homomorphism from $\mathscr{H}_{\kappa}$ onto $\mathcal{H}_{\tilde{\kappa}}$. By its definition, $f^{\prime}$ is inverse to $f$. Therefore $\mathscr{H}_{\kappa}$ and $\mathscr{H}_{\tilde{\kappa}}$ are isomorphic as filtered algebras. (Note that this isomorphism did not require that $\mathscr{H}_{\tilde{\kappa}}$ satisfy the PBW condition, only that $\tilde{\kappa}$ be a cocycle.) As gr $\mathscr{H}_{\kappa} \cong \mathrm{gr} \mathscr{H}_{\tilde{\kappa}} \cong S \# G$, the quotient algebra $\mathscr{H}_{\kappa}$ is also a Drinfeld 
orbifold algebra. Theorem 3.1 and Lemma 7.1 then imply the four conditions of the theorem.

Conversely, assume $\mathscr{H}_{\tilde{\kappa}}$ is isomorphic, as a filtered algebra, to some $\mathscr{H}_{\kappa}$ satisfying the four conditions of the theorem. Then Theorem 3.1 and Lemma 7.1 imply that $\mathscr{H}_{\kappa}$ is a Drinfeld orbifold algebra. As the isomorphism preserves the filtration,

$$
\operatorname{gr} \mathscr{H}_{\tilde{\kappa}} \cong \operatorname{gr} \mathscr{H}_{\kappa} \cong S(V) \# G
$$

as algebras, and hence $\mathscr{H}_{\tilde{\kappa}}$ is a Drinfeld orbifold algebra as well. Note that Theorem 6.3 shows that the bracket formula in the statement of the theorem indeed coincides with the Gerstenhaber bracket on cohomology.

Remark 7.3. We compare the above results to Gerstenhaber's original theory of deformations, since every Drinfeld orbifold algebra defines a deformation of $S \# G$ (see Section 2). The theory of Hochschild cohomology provides necessary conditions for "parameter maps" to define a deformation. Given a $k$-algebra $R$ and arbitrary $k$-linear maps $\mu_{1}, \mu_{2}: R \otimes R \rightarrow R$, we say $\mu_{1}$ and $\mu_{2}$ extend to first and second order approximations, respectively, of a deformation $R[t]$ of $R$ over $k[t]$ if there are $k$-linear maps $\mu_{i}: R \otimes R \rightarrow R(i \geq 3)$ for which the multiplication in $R[t]$ satisfies

$$
r * s=r s+\mu_{1}(r \otimes s) t+\mu_{2}(r \otimes s) t^{2}+\mu_{3}(r \otimes s) t^{3}+\cdots
$$

for all $r, s \in R$, where $r s$ is the product in $R$. Associativity forces $\mu_{1}$ to define a cocycle in $\mathrm{HH}^{2}(R)$; in addition, its Gerstenhaber square bracket must be twice the differential applied to $\mu_{2}$ :

$$
\left[\mu_{1}, \mu_{1}\right]=2 \delta_{3}^{*} \mu_{2} .
$$

Indeed, by using (2.3) and (2.4), we find that the equation $\left[\kappa^{L}, \kappa^{L}\right]=2 d^{*} \kappa^{C}$ is a consequence of the equation $\left[\mu_{1}, \mu_{1}\right]=2 \delta_{3}^{*} \mu_{2}$ : The left side of Theorem 3.1(iii) is both equal to $-\left[\kappa^{L}, \kappa^{L}\right]$ applied to $v_{1} \wedge v_{2} \wedge v_{3}$ and to $-\left[\mu_{1}, \mu_{1}\right]$ applied to $v_{1} \wedge v_{2} \wedge v_{3}$ by our previous analysis, identifying $\alpha$ in the Braverman-Gaitsgory approach with the restriction of $\mu_{1}$ to the space of relations $R$. The right side of (iii) is both equal to $-2 d^{*} \kappa^{C}$ applied to $v_{1} \wedge v_{2} \wedge v_{3}$ and to $-2 \delta_{3}^{*} \mu_{2}$ applied to $v_{1} \wedge v_{2} \wedge v_{3}$ since $\mu_{2} \circ \phi_{2}=\kappa^{C}$ and $\phi$ is a chain map (see (6.4)).

The square bracket $\left[\mu_{1}, \mu_{1}\right]$ is called the primary obstruction to integrating a map $\mu_{1}$ to a deformation: If a deformation exists with first-order approximation $\mu_{1}$, then $\left[\mu_{1}, \mu_{1}\right]$ is a coboundary, i.e., defines the zero cohomology class of the Hochschild cohomology $\mathrm{HH}^{3}(R)$.

The parameter maps $\kappa^{L}$ and $\kappa^{C}$ (arising from the Koszul resolution) play the role of the first and second order approximation maps $\mu_{1}$ and $\mu_{2}$ (arising from the bar complex). We see in the proof of Theorem 2.1 that each $\kappa_{g}^{L} g$ is in fact a cocycle when $\mathscr{H}_{\kappa}$ is a Drinfeld orbifold algebra, and each $\kappa_{g}^{C} g$ defines a second 
order approximation to the deformation. In fact, we expect $\kappa^{L}$ to be invariant whenever $\mathscr{H}_{\kappa}$ is a Drinfeld orbifold algebra since $\mathrm{HH}^{\bullet}(S \# G) \cong \mathrm{HH}^{\bullet}(S, S \# G)^{G}$. Note however that the theorem above goes beyond these elementary observations and Gerstenhaber's original formulation, which only give necessary conditions.

We now apply Theorem 7.2 in special cases to determine Drinfeld orbifold algebras from the set of necessary and sufficient conditions given in that theorem (in terms of Gerstenhaber brackets).

Recall that the Lie orbifold algebras are exactly the PBW algebras $\mathscr{H}_{\kappa}$ in which the linear part of the parameter $\kappa$ is supported on the identity group element $1_{G}$ alone. Interpreting Proposition 4.1 in homological language, we obtain necessary and sufficient conditions for $\kappa$ to define a Lie orbifold algebra in terms of the Gerstenhaber bracket:

Corollary 7.4. Assume $\kappa^{L}$ is supported on $1_{G}$. Then $\mathscr{H}_{\kappa}$ is a Lie orbifold algebra if and only if

(a) $\kappa^{L}$ is a Lie bracket on $V$,

(b) both $\kappa^{L}$ and $\kappa^{C}$ are $G$-invariant cocycles (define elements of $\mathrm{HH}^{2}(S \# G)$ ),

(c) $\left[\kappa^{C}, \kappa^{L}\right]=0$ as a cochain.

Proof. First note that $\left[\kappa^{L}, \kappa^{L}\right]=0$ exactly when $\kappa^{L}$ defines a Lie bracket on $V$. Suppose conditions (a), (b), and (c) hold. Condition (b) implies parts 1. and 2. of Theorem 7.2. It also implies that $d^{*}\left(\kappa^{C}\right)=0$. Condition (a) implies that $\left[\kappa^{L}, \kappa^{L}\right]=0$, and part 3. of Theorem 7.2 is satisfied as well. Condition (c) is part 4 . of Theorem 7.2. Hence, Theorem 7.2 implies that $\mathscr{H}_{\kappa}$ is a Lie orbifold algebra.

Conversely, assume that $\mathscr{H}_{\kappa}$ is a Lie orbifold algebra. By Proposition 4.1, $\kappa^{L}$ defines a Lie bracket on $V$ and hence $\left[\kappa^{L}, \kappa^{L}\right]=0$. Theorem 7.2 then not only implies condition (c), but also that $\kappa^{L}$ and $\kappa^{C}$ are both cocycles with $\kappa G$-invariant. But $\kappa$ is $G$-invariant if and only if both $\kappa^{L}, \kappa^{C}$ are $G$-invariant. Hence, condition (b) holds.

Recall that $\left(H^{\bullet}\right)^{G} \cong \mathrm{HH}^{\bullet}(S \# G)$ and that $C^{\bullet}$ and $H^{\bullet}$ are sets of cochains and cohomology representatives, respectively (see (5.4) and (5.5)). Given $\kappa^{C}, \kappa^{L}$ in $\left(H^{2}\right)^{G}$ of homogeneous polynomial degrees 0 and 1, respectively, the sum $\kappa:=$ $\kappa^{C}+\kappa^{L}$ is a parameter function $V \wedge V \rightarrow(k \oplus V) \otimes k G$ defining a quotient algebra $\mathscr{H}_{\kappa}$. The last result implies immediately that for $\kappa^{L}$ supported on $1_{G}$, the algebra $\mathscr{H}_{\kappa}$ is a Lie orbifold algebra when $\kappa^{L}$ is a Lie bracket on $V$ and the cochain $\left[\kappa^{C}, \kappa^{L}\right]$ is zero on the Koszul resolution. The hypothesis that $\kappa^{L}$ be a Lie bracket is not as restrictive as one might think. In fact, if $\kappa^{L}$ is a noncommutative Poisson structure (i.e., with Gerstenhaber square bracket $\left[\kappa^{L}, \kappa^{L}\right]$ zero in cohomology), then $\kappa^{L}$ is automatically a Lie bracket, as we see in the next corollary. 
Corollary 7.5. Suppose a linear cochain $\kappa^{L}$ in $C^{2}$ is supported on the kernel of the representation $G \rightarrow \mathrm{GL}(V)$ and that $\left[\kappa^{L}, \kappa^{L}\right]$ is a coboundary. Then $\left[\kappa^{L}, \kappa^{L}\right]=0$ as a cochain.

Proof. Suppose $\left[\kappa^{L}, \kappa^{L}\right]=d^{*} \alpha$ for some $\alpha$. Then (by definition of the map $d^{*}$ ),

$$
d_{3}^{*} \alpha\left(v_{1} \wedge v_{2} \wedge v_{3}\right)=-\sum_{g \in G} \sum_{\sigma \in \mathrm{Alt}_{3}} \alpha_{g}\left(v_{\sigma(2)}, v_{\sigma(3)}\right)\left({ }^{g} v_{\sigma(1)}-v_{\sigma(1)}\right) g
$$

for all $v_{1}, v_{2}, v_{3}$ in $V$, and thus $d^{*} \alpha$ is supported off the kernel $K$ of the representation $G \rightarrow \mathrm{GL}(V)$. But by Definition 5.6, $\left[\kappa^{L}, \kappa^{L}\right]$ is supported on $K$, since $\kappa^{L}$ itself is supported on $K$. Hence $\left[\kappa^{L}, \kappa^{L}\right]$ must be the zero cochain.

The last corollary implies that every linear noncommutative Poisson structure supported on group elements acting trivially lifts (or integrates) to a deformation of $S \# G$ :

Corollary 7.6. Suppose a linear cocycle $\kappa^{L}$ in $\left(H^{2}\right)^{G}$ has trivial Gerstenhaber square bracket in cohomology. If $\kappa^{L}$ is supported on the kernel of the representation $G \rightarrow \mathrm{GL}(V)$, then the quotient algebra $\mathscr{H}_{\kappa}$ with $\kappa=\kappa^{L}$ is a Drinfeld orbifold algebra. Moreover, if $G$ acts faithfully on $V$, then $\mathscr{H}_{\kappa} \cong \mathcal{U}(\mathfrak{g}) \# G$, a Lie orbifold algebra.

Proof. Since $\kappa^{L}$ lies in $\left(H^{2}\right)^{G}$, we may set $\kappa^{C} \equiv 0$ and $\kappa:=\kappa^{L}$ to satisfy the conditions of Theorem 7.2 (using Corollary 7.5 to deduce that $\kappa^{L}$ is a Lie bracket). If $G$ acts faithfully, the resulting Drinfeld orbifold algebra is just the skew group algebra $U(\mathfrak{g}) \# G$, where the Lie algebra $\mathfrak{g}$ is the vector space $V$ with Lie bracket $\kappa^{L}$.

Remark 7.7. The analysis of the Gerstenhaber bracket in [Shepler and Witherspoon 2012] includes information on the case of cocycles supported off the kernel $K$ of the representation $G \rightarrow \mathrm{GL}(V)$. Indeed, we see in that article that if $\kappa^{L}$ in $\left(H^{2}\right)^{G}$ is supported off $K$, then $\left[\kappa^{L}, \kappa^{L}\right]$ is always a coboundary. This guarantees existence of a constant cochain $\kappa^{C}$ with $\left[\kappa^{L}, \kappa^{L}\right]=2 d^{*} \kappa^{C}$. Thus to satisfy the conditions of Theorem 7.2, one need only check that $\left[\kappa^{C}, \kappa^{L}\right]=0$ as a cochain (on the Koszul resolution).

On the other hand, if $\kappa^{L}$ in $H^{\bullet}$ is supported on the kernel $K$, and $\left[\kappa^{L}, \kappa^{L}\right]$ is a coboundary, then by Corollary $7.5,\left[\kappa^{L}, \kappa^{L}\right]=0$ as a cochain. Thus to satisfy the conditions of Theorem 7.2, one need only solve the equation $\left[\kappa^{C}, \kappa^{L}\right]=0$ as a cochain for $\kappa^{C}$ a cocycle.

\section{Applications to abelian groups}

The last section expressed the PBW condition in terms of simple conditions on Hochschild cocycles. We see in this section how this alternative formulation gives 
a quick and clear proof that every linear noncommutative Poisson structure (i.e., Hochschild 2-cocycle with trivial Gerstenhaber square bracket) lifts to a deformation when $G$ is abelian. Halbout, Oudom, and Tang [Halbout et al. 2011, Theorem 3.7] gave an analogous result over the real numbers for arbitrary groups (acting faithfully), but their proof does not directly extend to other fields such as the complex numbers. (For example, complex reflections in a finite group acting linearly on $\mathbb{C}^{n}$ may contribute to Hochschild cohomology $\mathrm{HH}^{2}(S \# G)$ defined over the real numbers, but not to the same cohomology defined over the complex numbers.)

In the case of nonabelian groups, the square bracket of the linear part of the parameter $\kappa$ may be zero in cohomology but nonzero as a cochain. The following proposition explains that this complication disappears for abelian groups:

Proposition 8.1. Let $G$ be an abelian group. Let $\alpha, \beta$ in $\left(H^{2}\right)^{G}$ be linear with Gerstenhaber bracket $[\alpha, \beta]$ a coboundary (defining the zero cohomology class). Then $[\alpha, \beta]=0$ as a cochain.

Proof. Let $v_{1}, \ldots, v_{n}$ be a basis of $V$ on which $G$ acts diagonally. If $[\alpha, \beta]$ is nonzero at the chain level, then some summand of Definition 5.6 is nonzero for some triple $v_{1}, v_{2}, v_{3}$. Suppose without loss of generality that

$$
w=\beta_{h}\left(v_{3} \wedge \alpha_{g}\left(v_{1} \wedge v_{2}\right)\right)
$$

is nonzero for some $g, h$ in $G$.

Note that if $g$ acts nontrivially on $V$, then $v_{1}$ and $v_{2}$ must span $\left(V^{g}\right)^{\perp}$ and $\alpha_{g}\left(v_{1} \wedge v_{2}\right)$ lies in $V^{g}$ as $\alpha_{g}\left(v_{1} \wedge v_{2}\right)$ is nonzero and $\alpha_{g} \in H_{g}^{2}$. Similarly, if $h$ acts nontrivially on $V$, then $v_{3}$ and $\alpha_{g}\left(v_{1} \wedge v_{2}\right)$ must span $\left(V^{h}\right)^{\perp}$ and $w$ lies in $V^{h}$. (See the comments after (5.5) or [Shepler and Witherspoon 2008, Lemma 3.6].)

Suppose first that both $g$ and $h$ act nontrivially on $V$. Then $v_{3}$ and $\alpha_{g}\left(v_{1} \wedge v_{2}\right)$ are independent vectors in $V^{g} \cap\left(V^{h}\right)^{\perp}$, a subspace of the 2-dimensional space $\left(V^{h}\right)^{\perp}$. Thus $\left(V^{h}\right)^{\perp} \subset V^{g}$ and $v_{1}, v_{2}$ in $\left(V^{g}\right)^{\perp}$ are fixed by $h$. As $G$ is abelian and $\alpha$ is $G$-invariant, $\alpha_{g}={ }^{h} \alpha_{g}$ and

$$
\alpha_{g}\left(v_{1} \wedge v_{2}\right)=\left({ }^{h^{-1}} \alpha_{g}\right)\left(v_{1} \wedge v_{2}\right)={ }^{h^{-1}}\left(\alpha_{g}\left({ }^{h} v_{1} \wedge{ }^{h} v_{2}\right)\right)=h^{-1}\left(\alpha_{g}\left(v_{1} \wedge v_{2}\right)\right) .
$$

But then $\alpha_{g}\left(v_{1} \wedge v_{2}\right)$ is fixed by $h$, contradicting the fact that it lies in $\left(V^{h}\right)^{\perp}$.

We use the fact that the cochain map $[\alpha, \beta]$ represents the zero cohomology class to analyze the case when either $g$ or $h$ acts trivially on $V$. Calculations show that the image of the differential $d^{*}$ is supported on elements of $G$ that do not fix $V$ pointwise (see, for example, Section 7). Hence $V^{h g} \neq V$ and either $g$ or $h$ acts nontrivially on $V$. Also note that the coefficient of $g h$ in any image of the differential lies in $\left(V^{g h}\right)^{\perp}$.

If $h$ acts nontrivially on $V$ but $g$ fixes $V$ pointwise, then $w$ lies in $\left(V^{g h}\right)^{\perp}=\left(V^{h}\right)^{\perp}$, contradicting the fact that $w$ lies in $V^{h}$ (as $h$ acts nontrivially). If instead $g$ acts 
nontrivially on $V$ but $h$ fixes $V$ pointwise, we contradict the $G$-invariance of $\beta$ : In this case,

$$
\begin{aligned}
w & =\beta_{h}\left(v_{3} \wedge \alpha_{g}\left(v_{1} \wedge v_{2}\right)\right)=\left({ }^{g-1} \beta_{h}\right)\left(v_{3} \wedge \alpha_{g}\left(v_{1} \wedge v_{2}\right)\right) \\
& ={ }^{g-1}\left(\beta_{h}\left({ }^{g} v_{3} \wedge{ }^{g}\left(\alpha_{g}\left(v_{1} \wedge v_{2}\right)\right)\right)\right)={ }^{g^{-1}}\left(\beta_{h}\left(v_{3} \wedge\left(\alpha_{g}\left(v_{1} \wedge v_{2}\right)\right)\right)\right)={ }^{g^{-1}} w
\end{aligned}
$$

(since both $v_{3}$ and $\alpha_{g}\left(v_{1} \wedge v_{2}\right)$ lie in $\left.V^{g}\right)$, so $w$ lies in $V^{g}=V^{g h}$ instead of $\left(V^{g h}\right)^{\perp}$.

As a consequence of Lemma 7.1 and Proposition 8.1, we obtain the following:

Corollary 8.2. Let $G$ be an abelian group. Suppose $\kappa^{L}$ in $\left(H^{2}\right)^{G}$ is a linear cocycle with $\left[\kappa^{L}, \kappa^{L}\right]$ a coboundary. Then $\left[\kappa^{L}, \kappa^{L}\right]=0$ as a cochain. Thus we obtain a Drinfeld orbifold algebra $\mathscr{H}_{\kappa}$ after setting $\kappa^{C} \equiv 0$ and $\kappa:=\kappa^{L}$.

Other Drinfeld orbifold algebras with the same parameter $\kappa^{L}$ arise from solving the equation $\left[\kappa^{C}, \kappa^{L}\right]=0$ for $\kappa^{C}$ a cocycle of polynomial degree 0 in $\left(H^{2}\right)^{G}$. Compare with [Halbout et al. 2011, Theorem 3.4], which is stated in the case that the action is faithful.

We end this section by pointing out a much stronger statement than that implied by [Shepler and Witherspoon 2012, Theorem 9.2] for abelian groups: There we proved that for all groups $G$, the bracket of any two Hochschild 2-cocycles supported off the kernel of the representation is a coboundary (i.e., zero in cohomology). The proposition below (cf. [Halbout et al. 2011, Lemma 3.3]) explains that when $G$ is abelian, such brackets are not only coboundaries, they are zero as cochains.

Proposition 8.3. Let $G$ be an abelian group. Let $\alpha, \beta$ in $\left(H^{2}\right)^{G}$ be two linear Hochschild 2-cocycles on $S \# G$ supported off of the kernel of the representation $G \rightarrow \mathrm{GL}(V)$. Then $[\alpha, \beta]=0$ as a cochain.

Proof. This statement follows immediately from [Shepler and Witherspoon 2012, Theorem 9.2] and Proposition 8.1. However, we give a short, direct proof here: Let $v_{1}, \ldots, v_{n}$ be a basis of $V$ on which $G$ acts diagonally. If $[\alpha, \beta]$ is nonzero, then some summand

$$
\beta_{h}\left(v_{3} \wedge \alpha_{g}\left(v_{1} \wedge v_{2}\right)\right)
$$

of Definition 5.6 is nonzero for some triple $v_{1}, v_{2}, v_{3}$ in $V$ and some $g$ and $h$ in $G$. Since $g$ and $h$ both act nontrivially on $V$, the vector $\alpha_{g}\left(v_{1} \wedge v_{2}\right)$ must be invariant under $h$ (as we saw in the third paragraph of the proof of Proposition 8.1). But this contradicts the fact that $v_{3}$ and $\alpha_{g}\left(v_{1} \wedge v_{2}\right)$ must $\operatorname{span}\left(V^{h}\right)^{\perp}$.

One may apply Proposition 8.3 to find many examples of Drinfeld orbifold algebras of the type given in Example 3.3. 


\section{References}

[Beilinson et al. 1996] A. Beilinson, V. Ginzburg, and W. Soergel, "Koszul duality patterns in representation theory", J. Amer. Math. Soc. 9:2 (1996), 473-527. MR 96k:17010 Zbl 0864.17006

[Bergman 1978] G. M. Bergman, "The diamond lemma for ring theory", Adv. in Math. 29:2 (1978), 178-218. MR 81b:16001 Zbl 0326.16019

[Braverman and Gaitsgory 1996] A. Braverman and D. Gaitsgory, "Poincaré-Birkhoff-Witt theorem for quadratic algebras of Koszul type", J. Algebra 181:2 (1996), 315-328. MR 96m:16012 Zbl 0860.17002

[Bueso et al. 2003] J. Bueso, J. Gómez-Torrecillas, and A. Verschoren, Algorithmic methods in non-commutative algebra: Applications to quantum groups, Mathematical Modelling: Theory and Applications 17, Kluwer Academic, Dordrecht, 2003. MR 2005c:16069 Zbl 1063.16054

[Căldăraru et al. 2004] A. Căldăraru, A. Giaquinto, and S. Witherspoon, "Algebraic deformations arising from orbifolds with discrete torsion”, J. Pure Appl. Algebra 187:1-3 (2004), 51-70. MR 2005c:16013 Zbl 1055.16010

[Drinfeld 1986] V. G. Drinfeld, "Degenerate affine Hecke algebras and Yangians", Funct. Anal. Appl. 20:1 (1986), 58-60. Zbl 0599.20049

[Du et al. 2007] Y. Du, X. Chen, and Y. Ye, "On graded bialgebra deformations", Algebra Colloq. 14:2 (2007), 301-312. MR 2008a:16057 Zbl 1128.16023

[Etingof and Ginzburg 2002] P. Etingof and V. Ginzburg, "Symplectic reflection algebras, CalogeroMoser space, and deformed Harish-Chandra homomorphism", Invent. Math. 147:2 (2002), 243-348. MR 2003b:16021 Zbl 1061.16032

[Farinati 2005] M. Farinati, "Hochschild duality, localization, and smash products", J. Algebra 284:1 (2005), 415-434. MR 2005j:16009 Zbl 1066.16010

[Gerstenhaber 1963] M. Gerstenhaber, "The cohomology structure of an associative ring", Ann. of Math. (2) 78 (1963), 267-288. MR 28 \#5102 Zbl 0131.27302

[Ginzburg and Kaledin 2004] V. Ginzburg and D. Kaledin, "Poisson deformations of symplectic quotient singularities”, Adv. Math. 186:1 (2004), 1-57. MR 2005h:32072 Zbl 1062.53074

[Griffeth 2010] S. Griffeth, "Towards a combinatorial representation theory for the rational Cherednik algebra of type $G(r, p, n)$ ", Proc. Edinb. Math. Soc. (2) 53:2 (2010), 419-445. MR 2011f:16038 Zbl 1227.05265

[Halbout et al. 2011] G. Halbout, J.-M. Oudom, and X. Tang, "Deformations of orbifolds with noncommutative linear Poisson structures”, Int. Math. Res. Not. 2011:1 (2011), 1-39. MR 2012d:53284 Zbl 1207.53082

[Khare 2007] A. Khare, "Drinfeld-Hecke algebras over cocommutative algebras", preprint, 2007. arXiv 0705.2067

[Levandovskyy and Shepler 2011] V. Levandovskyy and A. V. Shepler, "Quantum Drinfeld Hecke algebras", preprint, 2011. arXiv 1111.4975

[Lusztig 1988] G. Lusztig, "Cuspidal local systems and graded Hecke algebras, I", Inst. Hautes Études Sci. Publ. Math. 67 (1988), 145-202. MR 90e:22029 Zbl 0699.22026

[Lusztig 1989] G. Lusztig, "Affine Hecke algebras and their graded version", J. Amer. Math. Soc. 2:3 (1989), 599-635. MR 90e:16049 Zbl 0715.22020

[Ram and Shepler 2003] A. Ram and A. V. Shepler, "Classification of graded Hecke algebras for complex reflection groups", Comment. Math. Helv. 78:2 (2003), 308-334. MR 2004d:20007 Zbl 1063.20005 
[Shepler and Witherspoon 2008] A. V. Shepler and S. Witherspoon, "Hochschild cohomology and graded Hecke algebras", Trans. Amer. Math. Soc. 360:8 (2008), 3975-4005. MR 2009a:16015 Zbl 1195.20005

[Shepler and Witherspoon 2011] A. V. Shepler and S. Witherspoon, "Quantum differentiation and chain maps of bimodule complexes", Algebra Number Theory 5:3 (2011), 339-360. MR 2833794 Zbl 06000308

[Shepler and Witherspoon 2012] A. V. Shepler and S. Witherspoon, "Group actions on algebras and the graded Lie structure of Hochschild cohomology”, J. Algebra 351 (2012), 350-381. MR 2862214 Zbl 06046981

[Ştefan 1995] D. Ştefan, "Hochschild cohomology on Hopf Galois extensions", J. Pure Appl. Algebra 103:2 (1995), 221-233. MR 96h:16013 Zbl 1227.05265

[Weibel 1994] C. A. Weibel, An introduction to homological algebra, Cambridge Studies in Advanced Mathematics 38, Cambridge University Press, 1994. MR 95f:18001 Zbl 0797.18001

[Witherspoon 2007] S. Witherspoon, "Twisted graded Hecke algebras", J. Algebra 317:1 (2007), 30-42. MR 2009a:20009 Zbl 1139.20005

Received November 30, 2011. Revised July 9, 2012.

\author{
ANNE V. SHEPLER \\ DEPARTMENT OF MATHEMATICS \\ UNIVERSITY OF NORTH TEXAS \\ 1155 UNION CIRCLE \#311430 \\ DENTON, TX 76203-5017 \\ UNITED STATES \\ ashepler@unt.edu \\ SARAH WITHERSPOON \\ DEPARTMENT OF MATHEMATICS \\ TEXAS A\&M UNIVERSITY \\ MAILSTOP 3368 \\ College Station, TX 77843 \\ UNITED STATES \\ sjw@math.tamu.edu
}




\title{
PACIFIC JOURNAL OF MATHEMATICS
}

\author{
http://pacificmath.org \\ Founded in 1951 by \\ E. F. Beckenbach (1906-1982) and F. Wolf (1904-1989)
}

\section{EDITORS}

V. S. Varadarajan (Managing Editor)

Department of Mathematics

University of California

Los Angeles, CA 90095-1555

pacific@math.ucla.edu

Vyjayanthi Chari

Department of Mathematics

University of California

Riverside, CA 92521-0135

chari@math.ucr.edu

\section{Robert Finn}

Department of Mathematics Stanford University

Stanford, CA 94305-2125

finn@math.stanford.edu

Kefeng Liu

Department of Mathematics

University of California

Los Angeles, CA 90095-1555

liu@math.ucla.edu
Darren Long

Department of Mathematics

University of California

Santa Barbara, CA 93106-3080

long@math.ucsb.edu

Jiang-Hua Lu

Department of Mathematics

The University of Hong Kong

Pokfulam Rd., Hong Kong jhlu@maths.hku.hk

Alexander Merkurjev

Department of Mathematics

University of California

Los Angeles, CA 90095-1555

merkurev@math.ucla.edu
Sorin Popa

Department of Mathematics University of California

Los Angeles, CA 90095-1555 popa@math.ucla.edu

Jie Qing

Department of Mathematics

University of California

Santa Cruz, CA 95064

qing@cats.ucsc.edu

Jonathan Rogawski

Department of Mathematics

University of California

Los Angeles, CA 90095-1555

jonr@math.ucla.edu

\section{PRODUCTION}

pacific@math.berkeley.edu

\section{SUPPORTING INSTITUTIONS}

ACADEMIA SINICA, TAIPEI

CALIFORNIA INST. OF TECHNOLOGY INST. DE MATEMÁTICA PURA E APLICADA KEIO UNIVERSITY

MATH. SCIENCES RESEARCH INSTITUTE NEW MEXICO STATE UNIV.

OREGON STATE UNIV.

\author{
STANFORD UNIVERSITY \\ UNIV. OF BRITISH COLUMBIA \\ UNIV. OF CALIFORNIA, BERKELEY \\ UNIV. OF CALIFORNIA, DAVIS \\ UNIV. OF CALIFORNIA, LOS ANGELES \\ UNIV. OF CALIFORNIA, RIVERSIDE \\ UNIV. OF CALIFORNIA, SAN DIEGO \\ UNIV. OF CALIF., SANTA BARBARA
}

\author{
UNIV. OF CALIF., SANTA CRUZ \\ UNIV. OF MONTANA \\ UNIV. OF OREGON \\ UNIV. OF SOUTHERN CALIFORNIA \\ UNIV. OF UTAH \\ UNIV. OF WASHINGTON \\ WASHINGTON STATE UNIVERSITY
}

These supporting institutions contribute to the cost of publication of this Journal, but they are not owners or publishers and have no responsibility for its contents or policies.

See inside back cover or pacificmath.org for submission instructions.

The subscription price for 2012 is US \$420/year for the electronic version, and \$485/year for print and electronic.

Subscriptions, requests for back issues from the last three years and changes of subscribers address should be sent to Pacific Journal of Mathematics, P.O. Box 4163, Berkeley, CA 94704-0163, U.S.A. Prior back issues are obtainable from Periodicals Service Company, 11 Main Street, Germantown, NY 12526-5635. The Pacific Journal of Mathematics is indexed by Mathematical Reviews, Zentralblatt MATH, PASCAL CNRS Index, Referativnyi Zhurnal, Current Mathematical Publications and the Science Citation Index.

The Pacific Journal of Mathematics (ISSN 0030-8730) at the University of California, c/o Department of Mathematics, 969 Evans Hall, Berkeley, CA 94720-3840, is published monthly except July and August. Periodical rate postage paid at Berkeley, CA 94704, and additional mailing offices. POSTMASTER: send address changes to Pacific Journal of Mathematics, P.O. Box 4163, Berkeley, CA 94704-0163.

PJM peer review and production are managed by EditFLOW ${ }^{\mathrm{TM}}$ from Mathematical Sciences Publishers.

PUBLISHED BY PACIFIC JOURNAL OF MATHEMATICS

at the University of California, Berkeley 94720-3840

A NON-PROFIT CORPORATION

Typeset in LATEX

Copyright $(02012$ by Pacific Journal of Mathematics 


\title{
PACIFIC JOURNAL OF MATHEMATICS
}

\author{
Volume $259 \quad$ No. $1 \quad$ September 2012
}

Extension Theorems for external cusps with minimal regularity

GABRIEL ACOSTA and IGNACIO OJEA

Convergence of axially symmetric volume-preserving mean curvature flow

MARIA ATHANASSENAS and SEVVANDi KANDANAARACHCHI

On the horoboundary and the geometry of rays of negatively curved manifolds

FranÇOISE DAL'bo, Marc PEIGNÉ and ANDREA

SAMBUSETTI

Two infinite versions of the nonlinear Dvoretzky theorem

KEI FUNANO

Nonlocal uniform algebras on three-manifolds

ALEXANDER J. IZZO

Mahlo cardinals and the torsion product of primary abelian groups

PATRICK W. KEEF

Geometry of trinomials

AARON MELMAN

Drinfeld orbifold algebras

ANNE V. SHEPLER and SARAH Witherspoon

Semi-topological cycle theory I

JYH-HAUR TEH

New construction of fundamental domains for certain Mostow groups 209

TiEHONG ZHAO 